\title{
Treatment of mouse liver slices with cholestatic hepatotoxicants results in down-regulation of Fxr and its target genes
}

\author{
Ewa Szalowska*, Geert Stoopen, Maria J Groot, Peter JM Hendriksen and Ad ACM Peijnenburg
}

\begin{abstract}
Background: Unexpected cholestasis substantially contributes to drug failure in clinical trials. Current models used for safety assessment in drug development do not accurately predict cholestasis in humans. Therefore, it is of relevance to develop new screening models that allow identifying drugs with cholestatic properties.

Methods: We employed mouse precision cut liver slices (PCLS), which were incubated $24 \mathrm{~h}$ with two model cholestatic compounds: cyclosporin A (CsA) and chlorpromazine (CPZ). Subsequently, transcriptome analysis using DNA microarrays and q-PCR were performed to identify relevant biological processes and biomarkers. Additionally, histology was carried out and levels of triglycerides (TG) and bile acids (BA) were measured. To verify the ex vivo mouse data, these were compared with publically available human data relevant for cholestasis.

Results: Whole genome gene expression analysis showed that CSA up-regulated pathways related to NF-KB, ER stress and inflammation. Both CSA and CPZ down-regulated processes related to extracellular matrix (ECM) remodelling, BA homeostasis, Fxr signalling, and energy metabolism. The differential expression of a number of characteristic genes (e.g. Abcg5, Abcg8, Klf15, and Baat) could be confirmed by q-PCR. Histology revealed that CsA but not CPZ induced "ballooning" of hepatocytes. No effects on TG and BA levels were observed after incubation of PCLS with CSA and CPZ. A substantial number of processes altered in CSA- and CPZ-treated mouse PCLS ex vivo was also found to be affected in liver biopsies of cholestatic patients.

Conclusion: The present study demonstrated that mouse PCLS can be used as a tool to identify mechanisms of action of cholestatic model compounds. The induction of general stress responses and down-regulated Fxr signalling could play a role in the development of drug induced cholestasis. Importantly, comparative data analysis showed that the ex vivo mouse findings are also relevant for human pathology. Moreover, this work provides a set of genes that are potentially useful to assess drugs for cholestatic properties.
\end{abstract}

Keywords: Cholestasis, Precision cut liver slices, Alternatives for animal testing, Transcriptome, Fxr (Nr1h4), Biomarkers

\section{Background}

Drug induced liver injury is a major problem in medical care and pharmacological industry. One example of severe liver damage is cholestasis that comprises approximately $17 \%$ of all hepatic adverse drug reactions [1]. Cholestasis results in a dramatic increase in liver and serum bile acids that eventually can lead to liver failure [2]. Several currently used drugs have been shown to induce cholestasis e.g. cyclosporin A (CsA) used as an immunosuppressant is

\footnotetext{
* Correspondence: ewa.szalowska@wur.nl

RIKILT - Institute of Food Safety, Wageningen UR, P.O. Box 230, 6700 AE, Wageningen, the Netherlands
}

known to induce cholestasis in human, rat and mouse [3,4], and chlorpromazine (CPZ) applied as an antipsychotic was shown to induce cholestasis in both human and rat [5]. It is likely that $\mathrm{CPZ}$ can also induce cholestasis in mouse but so far no studies addressing this issue have been reported. Bile acids (BA) are amphipathic molecules synthesized from cholesterol in hepatocytes, which together with phospholipids and cholesterol are actively secreted by the liver as bile. Upon a meal ingestion bile is released into the intestinal lumen to enable absorption of dietary lipids and fat-soluble vitamins [2]. BA levels are tightly controlled because high BA concentrations may become cytotoxic [2]. 
The nuclear receptor farnesoid X receptor (FXR/NR1H4, in the remaining part of the manuscript FXR symbol will be used) is considered as a master regulator of BA homeostasis. FXR upon BA binding regulates expression of several genes involved in BA homeostasis in the liver [2]. Impairment of FXR signaling and its downstream target genes can result in intrahepatic cholestasis. For example, dysregulation or mutations in genes encoding for bile salt export pump (BSEP), multidrug resistance protein 3 (MDR3) [6], or cholesterol 7a-hydroxylase (CYP7A1) [7] and bile acid-CoA:amino acid $\mathrm{N}$-acyltransferease (BAAT) can evoke cholestasis [8]. Other nuclear receptors (NRs), such as pregnane $X$ receptor (PXR), vitamin D $(1,25$ dihydroxyvitamin D3) receptor (VDR), and constitutive androstane receptor (CAR) are known to be involved in BA detoxification by regulating expression of genes involved in phase I and II reactions [9]. Moreover, FXR also plays a major role in regulation of lipid and glucose metabolism [2]. Therefore, FXR antagonism does not only induce cholestasis but also leads to increased accumulation of hepatic triglycerides [2]. For that reason, activation of FXR is used in the treatment of cholestasis and nonalcoholic steatohepatitis (NASH) [9].

Consistent with the fact that FXR is involved in both BA and lipid metabolism, drug induced cholestasis can be accompanied with the development of fatty liver diseases [10]. It has also been postulated that drug-induced cholestasis might lead to lipid accumulation as a secondary event of endoplasmatic reticulum (ER) stress causing mitochondrial dysfunction [11]. Additionally, inflammation mediated by Kupffer cells and $\mathrm{T}$ cells can contribute to progression of cholestasis [12]. Due to the complexity of BA homeostasis and involvement of different cell types in the development of cholestasis, it is difficult to study this process in vitro. Precision cut liver slices (PCLS) are commonly used as an in vitro/ex vivo liver model. The major advantage of PCLS is that they contain native liver architecture and all cell types characteristic for the liver in vivo [13]. Additionally, it has been shown that pathways mediated by FXR, VDR and PXR are also active in rat and human PCLS, therefore supporting the use of PCLS to study BA metabolism [13]. It has been reported that studies employing toxicogenomics in PCLS can correctly predict the toxicity and pathology observed in vivo [14].

The main goal of the present study was to assess whether application of mouse PCLS in combination with whole genome gene expression profiling is an useful approach to identify mechanisms leading to cholestasis. In addition, we aimed to identify genes that could serve as biomarkers to distinguish between cholestasis and other types of hepatotoxicity such as necrosis and steatosis in mouse PCLS. To that end, mouse PCLS were treated for 24 h with two known cholestatic drugs, CsA and CPZ, and subsequently subjected to DNA microarray analysis. Transcriptome analysis was combined with biochemical and histological examinations to allow comparison of the array data with classical parameters of cholestasis.

\section{Methods \\ Chemicals}

Cyclosporin A, chlorpromazine, valproic acid, amiodarone, paraquat, and isoniazid were purchased from Sigma (Zwijndrecht, the Netherlands). Williams E medium supplemented with Glutamax, penicillin/ streptomycin (pen/strep), D-Glucose, PBS were obtained from Invitrogen (Bleiswijk, the Netherlands).

\section{Preparation and culture of liver slices}

23-weeks old male C57BL/6 mice were obtained from Harlan (Horst, The Netherlands). Animals were kept for 1 week at a housing temperature of $22^{\circ} \mathrm{C}$ and at a relative humidity of $30-70 \%$. The lighting cycle was 12 -h light and 12-h dark. At the age of 24 weeks animals were sacrificed by an overdose of isoflurane. The treatment protocol was approved by the Ethical Committee for Animal Experiments at Wageningen University.

Immediately after the animals were killed the liver was perfused with PBS and placed in ice-cold Krebs-Henseleit buffer (KHB) (pH 7.4, supplemented with $11 \mathrm{mM}$ glucose). Liver tissue was transported to the laboratory within approximately $30 \mathrm{~min}$ and cylindrical liver cores were produced using a surgical biopsy punch with diameter of $5 \mathrm{~mm}$ (KAI, SynErgo Europe, Romania). Liver cores were placed in a Krumdieck tissue slicer (Alabama Research and Development, Munford, AL) filled with ice-cold KHB aerated with carbogen and supplemented with $11 \mathrm{mM}$ glucose. Slices with a diameter of $5 \mathrm{~mm}$, a thickness of $0.2 \mathrm{~mm}$ and a weight of approximately $6 \mathrm{mg}$ were prepared. Immediately after preparation, slices were transferred into culture plates filled with pre-warmed $\left(37^{\circ} \mathrm{C}\right)$ Williams E medium (WEM) supplemented with pen/strep. Three liver slices were pre-cultured in one well of the 6-well plate filled with $4 \mathrm{ml}$ of WEM for one hour with continuous shaking $(70 \mathrm{rpm})$. Incubations were performed in an oxygen controlled incubator (Galaxy $48 \mathrm{R}$ New Brunswick, Nijmegen, the Netherlands) at $80 \%$ of oxygen; $5 \% \mathrm{CO}_{2}$ and the remaining gas volume was filled up to $95 \%$ with $\mathrm{N}_{2}$. After one hour of pre-incubation, media were removed and replaced with fresh media containing test compounds or appropriate solvents. After incubations, samples were snap-frozen in liquid nitrogen and stored in $-80^{\circ} \mathrm{C}$ for further analysis. Samples dedicated to histology were stored in $4 \%$ formaldehyde at room temperature.

\section{Cytotoxicity analysis (dose selection)}

PCLS were exposed to different model compounds inducing cholestasis, steatosis, necrosis, and to compounds 
that are not hepatotoxic in vivo. These compounds were selected based on literature search. As cholestatic model compounds cyclosporin A (CsA), and chlorpromazine (CPZ) were selected. For steatosis valproic acid [15] and amiodarone [16] were selected. As pro-necrotic drugs paraquat [17] and isoniazid [18] were used. To select a non-toxic dose eventually to be used for the gene expression profiling experiments, the following concentrations ranges were tested: cyclosporin A $0-100 \mu \mathrm{M}$, chlorpromazine $0-80 \mu \mathrm{M}$, valproic acid 0-500 $\mu \mathrm{M}$, amiodarone $0-100$ $\mu \mathrm{M}$, paraquat $0-10 \mu \mathrm{M}$, and isoniazid $0-1000 \mu \mathrm{M}$, CyclosporinA, chlorpromazine, amiodarone, paraquat were dissolved in DMSO, valproic acid was dissolved in ethanol $(\mathrm{EtOH})$, and isoniazid was dissolved in PBS. The compounds were added to the culture medium at a final concentration of $0.1 \% \mathrm{vol} / \mathrm{vol}$ in an appropriate solvent (DMSO, EtOH, or PBS). Slices incubated with the solvents at $0.1 \% \mathrm{vol} / \mathrm{vol}$ served as controls. Toxicity was assessed with standard biochemical assays indicative for slices viability: LDH, ATP, and protein assay. Dose selection experiments were performed in slices obtained from at least two mice (see also Additional file 1: Figure S1, Additional file 2: Figure S2 and Additional file 3: Figure S3).

\section{ATP and protein measurements}

For each ATP and protein measurement a total of three co-cultured slices were placed in $400 \mu \mathrm{L}$ Cell Lytic MT buffer (Sigma, Zwijndrecht, the Netherlands). In order to compare ATP and protein content in liver slices from different experiments, both ATP and protein were normalized on mass of liver slices. In order to determine the weight of the liver slices, three liver slices were removed from the culture well. Thereafter, the three liver slices were gently held in tweezers against a paper tissue for 2 seconds to let the tissue absorb the remaining medium. Next, the three slices were weighed on a regular laboratory balance and the mass was registered for each individual exposure, which was $18 \mathrm{mg} \pm 25 \%$ for three slices. Slices were homogenized $\left(6500 \mathrm{~g}, 8^{\circ} \mathrm{C}\right)$ two times for $15 \mathrm{sec}$ using a tissue homogenizer (Precellys 24 Bertin Technologies, Labmakelaar Benelux B.V. Rotterdam, The Netherlands). To remove cellular debris, the homogenates were centrifuged for $5 \min \left(14000 \mathrm{~g}, 8^{\circ} \mathrm{C}\right)$ and the remaining supernatant was divided into two portions of $200 \mu \mathrm{L}$. One portion was stored at $-80^{\circ} \mathrm{C}$ for protein measurements and the second $200 \mu \mathrm{L}$ portion was mixed with $100 \mu \mathrm{L}$ of ATP lytic buffer (ATPlite, Perkin Elmer, Oosterhout, The Netherlands) for ATP measurements. ATP was measured according to the manufacturer's description using a microplate reader (Synergy TM HT Multi Detection Microplate Reader, Biotek Instruments Inc, Abcoude, the Netherlands) with settings for luminescence: 590/635 nm, top measurement, and sensitivity 230. ATP determination was performed in technical duplicates and luminescence values were recalculated into $\mu \mathrm{M}$ ATP in total liver slices extracts. ATP concentration was normalized on mass of liver slices used for one ATP extraction.

The protein concentration was determined according to the Bradford method (Protein assay, BioRad, Veenendaal, The Netherlands). Protein samples of $2 \mu \mathrm{L}$ were diluted 80 times in PBS and measured according to the manufacturer's instructions. BSA was used as a standard and each measurement was performed in duplicate. The protein content was normalized on mass of liver slices used for protein extraction.

\section{Lactate dehydrogenase (LDH) assay}

Lactate dehydrogenase activity in total tissue homogenate and in the culture medium of each slice was performed according to the manufacturer's protocol (Cytotoxicity Detection Kit (LDH), Roche Diagnostics, Almere, the Netherlands). For the total LDH activity in slices, three slices were homogenized $\left(6500 \mathrm{~g}, 8^{\circ} \mathrm{C}\right)$ twice for $15 \mathrm{sec}$ using a tissue homogenizer (Precellys 24). Thereafter, culture medium or the slice homogenate were diluted 5 fold with PBS and mixed with $100 \mu \mathrm{L}$ reaction mixture from the kit. After 15 minutes of incubation at room temperature, samples were measured at $490 \mathrm{~nm}$ using a Synergy TM HT Multi Detection microplate reader.

\section{Triglycerides (TG) measurements}

Three liver slices cultured together were used to make a homogenate in $300 \mu \mathrm{L}$ buffer containing $10 \mathrm{mM}$ Tris, $1 \mathrm{mM}$ EDTA (pH 7.4) and $250 \mathrm{mM}$ sucrose. Aliquots of $10 \mu \mathrm{L}$ of the liver slices homogenates were used in the assay and measurements were performed according to the manufacturer's protocol (triglycerides liquicolor mono, Instruchemie, Delfzijl, the Netherlands).

\section{Bile acids measurements}

For bile acids measurements, three slices were pooled and placed in $400 \mu \mathrm{L}$ Cell Lytic MT buffer (Sigma, Zwijndrecht, the Netherlands). Slices were homogenized for two times $15 \mathrm{sec}\left(6500 \mathrm{~g}, 8^{\circ} \mathrm{C}\right)$ using a tissue homogenizer (Precellys 24). Thereafter, the homogenates were centrifuged for $5 \mathrm{~min}\left(14000 \mathrm{~g}, 8^{\circ} \mathrm{C}\right)$ to remove cellular debris, and the supernatant was used for measurements. Bile acids quantification was performed according to the description in manufacturer's protocol (Total Bile Acid Assay Kit Bioquant, Gentaur, Brussels, Belgium).

\section{Histology}

Slices were exposed for 24 and 48 hours to $40 \mu \mathrm{M}$ CsA or $20 \mathrm{uM} \mathrm{CPZ}$. For the 48 hours cultures, after 24 hours the culture medium was replaced with fresh medium. Immediately after incubation, slices were fixed in $4 \%$ buffered formaldehyde. After embedding in paraffin, crosssections were prepared, stained with haematoxylin and 
eosin (HE) according to Mayer using standard procedures, and examined under a microscope with a $100 \mathrm{x}$ magnification [19]. Fouchet staining for bile acids pigments and Periodic acid-Schiff (PAS) for glycogen were performed according to standard protocols (http://www.ihcworld. com/histology.htm). For each condition slices obtained from 5 mice were analysed. All pictures were taken using 100-fold magnification.

\section{PCLS exposure (gene expression profiling)}

To obtain RNA for transcriptome analysis, PCLS were cultured at the same conditions as described above. Slices were exposed for 24 hours to one concentration of the tested compounds or controls. Concentrations used in the exposure experiments were pre-selected in the dose selection experiments (cytotoxicity analysis). The highest concentration that did not cause significant toxicity assessed by biochemical assays (LDH, ATP, and protein assay) was selected. The concentrations used in the exposure experiments were as follows; for the cholestatic exposures $40 \mu \mathrm{M}$ CsA and $20 \mu \mathrm{M} \mathrm{CPZ}$. For the steatogenic exposures: $200 \mu \mathrm{M}$ valproic acid, and $50 \mu \mathrm{M}$ amiodarone. For the necrotic compounds: $5 \mu \mathrm{M}$ paraquat and $1000 \mu \mathrm{M}$ isoniazid. Liver slices obtained from five mice were used in five separate experiments in which exposures to toxic compound or vehicle control were performed at the same time. Since dose selection experiments and exposure experiments were performed at different days, ATP, LDH, and protein assays were performed again for the exposure experiments to confirm that the selected doses did also not affect slice viability in these experiments (Figure 1).

\section{DNA microarray hybridizations}

Gene expression analysis in liver slices incubated for 24 hours with the cholestatic, steatogenic, and necrotic compounds as well as the controls was performed on the HT Mouse Genome 430 PM array plate(s) using the Affymetrix GeneTitan system. RNA was extracted from three slices cultured and exposed together using the RNeasy Tissue Mini Kit (Qiagen, Venlo, The Netherlands) according to the manufacturer's instructions. RNA concentration and purity were assessed spectrometrically using a Nano Drop ND-1000 spectrophotometer (Isogen IJsselstein, The Netherlands) by measuring absorption ratios at $260 / 280$ and $260 / 230 \mathrm{~nm}$. The integrity of the RNA samples was examined using the Shimadzu MultiNA Bioanalyzer (Tokyo, Japan). Biotin- labelled cRNA was generated from high-quality total RNA with the Affymetrix 3'IVT Express Kit (Affymetrix, Santa Clara, CA, USA) according to the manufacturer's specifications with an input of $100 \mathrm{ng}$ total RNA. The Agilent Bioanalyzer (Amstelveen, the Netherlands) and Shimadzu MultiNA Bioanalyzer (Tokyo, Japan) were used to evaluate the quality of cRNA in order to confirm if the average fragment size was according to Affymetrix' specifications. Per sample, 7.5ug cRNA of the obtained biotinylated cRNA samples was fragmented and hybridized in a final concentration of $0.0375 \mathrm{ug} / \mathrm{ul}$ on the Affymetrix HT Mouse genome 430 PM array (Affymetrix, Santa Clara, CA, USA. After an automated process of washing and staining by the GeneTitan machine (Affymetrix, Santa Clara, CA, USA) using the Affymetrix HWS kit for Gene Titan, absolute values of expression were calculated from the scanned array using the Affymetrix Command Console v 3.2 software. The data Quality Control was performed in the program Affymetix Expression Console v 1.1 software to determine if all parameters were within quality specifications. The Probe Logarithmic Intensity Error Estimation (PLIER) algorithm method was used for probe summarisation [20].

In order to monitor the sample independent control and the performance of each individual sample during hybridization, hybridizations controls were added to the hybridization mixture. The sample dependent controls such as internal control genes, background values, and average signals were used to determine the biological variation between samples. In conclusion, all the data were within data Quality Control thresholds, according to Affymetrix Expression Console specifications. Nonnormalized data in a form of the Cell Intensity File (".CEL) were re-annotated (EntrezGene htmg430pm Mm_ENTREZG) and the data were RMA normalized $[20,21]$.

\section{Gene Set Enrichment Analysis (GSEA)}

In order to identify differentially expressed gene sets related to diverse biological functions Gene Set Enrichment Analysis (GSEA) was applied. GSEA was performed using an open access bioinformatics tool (http://www.broadinstitute. org/gsea/index.jsp). In short, this method identifies biologically and functionally related genes affected due to experimental conditions. GSEA applies predefined gene sets that are based on literature or other experiments. Gene sets specify a group of genes specific for a certain biological process, GO ontology, pathway, or user defined groups. GSEA ranks all the genes on their expression ratios between a treatment and the control group and determines whether a particular gene set is significantly enriched at the top or the bottom of the ranked list [22]. Gene sets used in this study were created in an open access bioinformatics tool ANNI http://www.biosemantics.org/index. php?page=ANNI-2-0 [23]. ANNI retrieves all the information available on known gene-gene associations present in Medline and can be used, among others, to create gene sets associated with simple queries, for example "inflammation" or "cholestasis". For the purpose of this study we used several queries related to liver specific and liver nonspecific processes. Summary of all the queries used for the 


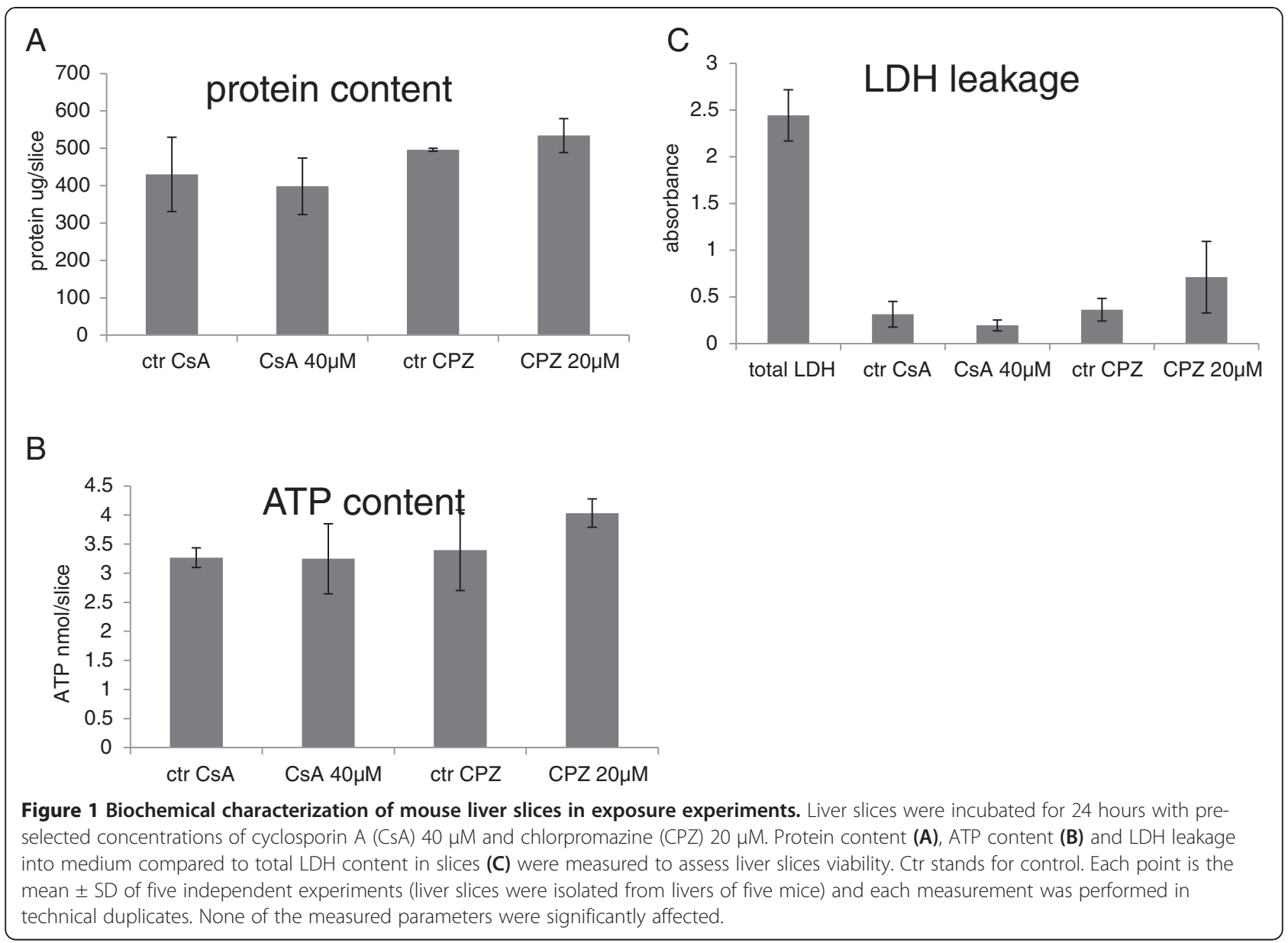

creation of "the ANNI gene sets" is given in Additional file 4: Table S1. Genes present in at least five publications indicating an association with the specified queries were included in the ANNI gene sets.

For GSEA, also Gene Expression Omnibus microarray data relevant for human cholestasis were used (GSE46960). These gene expression data were generated in GeneChip ${ }^{\circ}$ Human Gene 1.0 ST array (Affymetrix, CA) hybridisation experiments using human liver biopsies obtained from 64 infants with biliary atresia, 14 age-matched infants with cholestasis of other origin than biliary atresia, and from 7 deceased-donor healthy children.

\section{MetaCore pathway analysis}

The GSEA report output file informs which gene sets are significantly affected in the analysed groups. Additionally, it informs, which genes significantly contribute to this enrichment (significant genes). These significant genes were used as an input for the MetaCore pathway analysis. The pathway analysis was performed using Functional Ontology Enrichment/ Pathways Maps default option in MetaCore for mouse or human depending whether mouse or human data were analysed, respectively. MetaCore identifies pathways using a default enrichment analysis. The identified pathways were considered as significantly affected by the treatment if $\mathrm{p}<0.005$.

\section{Biomarker identification}

In order to identify biomarkers indicative for the development of cholestasis, significant genes identified in GSEA were subjected to Venn analysis. Genes overlapping between CsA and CPZ treatments with absolute FC $\geq 1.5$ were selected as potential biomarkers. Venn analysis was performed using an open access tool http://bioinfogp.cnb. csic.es/tools/venny/. Subsequently, biomarkers' expression values (derived from the DNA microarray data) were compared with expression values for the same genes in slices treated with non-cholestatic drugs. In order to do so, the gene expression values were $\log 2$ transformed and median centered followed by hierarchical clustering using default options in Genesis (http://genome.tugraz. at/genesisserver/genesisserver_description.shtml). Additionally, the candidate biomarkers were uploaded to another open access bioinformatics tool denoted with Search Tool for the Retrieval of Interacting Genes/Proteins 8.2 (STRING) in order to perform functional clustering. 
STRING identifies and visualizes functional networks based on known and predicted protein-protein interactions [24]. Functional clustering of genes was performed in STRING using functional clustering function.

\section{q-PCR}

In further studies, expression of ten candidate biomarkers in PCLS was analyzed by q-PCR using the Biorad CFX96 TM Real-Time Detection System (Bio-Rad, Veenendaal, The Netherlands) with the following cycling conditions: $15 \mathrm{~min} 95^{\circ} \mathrm{C}$ followed by 40 cycles of $15 \mathrm{~s} 95^{\circ} \mathrm{C}$ and $1 \mathrm{~min}$ $60^{\circ} \mathrm{C}$. Reactions were performed in $10 \mu \mathrm{l}$ and contained 20 ng cDNA, 1x TaqMan PCR Master Mix (Applied Biosystems, Foster City, CA), and depending on the analysed gene, $250 \mathrm{nM}$ probe and $900 \mathrm{nM}$ primer (for actin b) or 1xTaqMan gene expression assay (for the remaining genes) (Applied Biosystems, foster City, CA). Specific primer set for actin $\beta$ (ACTB) was developed with Primer Express 1.5 (Applied Biosystems, Nieuwerkerk aan den IJssel, The Netherlands) and sequences were as follows, probe: TGT CCC TGT ATG CCT CTG GTC GTA CCA C, forward primer: AGC CAT GTA CGT AGC CAT CCA, and reverse primer: TCT CCG GAG TCC ATC ACA ATG. Primers for Kruppel-like factor 15 (Klf15), bile acid-CoenzymeA:amino acid $\mathrm{N}$-acyltransferase (Baat), ATP-binding cassette, sub-family G (WHITE), member 8 (Abcg8), ATP-binding cassette, and sub-family G (WHITE), member 5 (Abcg5), were purchased from Applied Biosystems. The numbers of assays from Applied Biosystems are given in Additional file 5: Table S2. Data were analyzed with SDS 2.0 software (Bio-Rad). For each sample, the RT-PCR reaction was performed in triplicate and the averages of the obtained threshold cycle values $\left(C_{T}\right)$ were processed for further calculations. To check for contaminating DNA, samples without reverse transcriptase (-RT reaction) were analysed as well. For normalization ACTB was used. Relative expression was calculated with $\Delta$ $\left(\Delta\left(C_{\mathrm{T}}\right)\right)$-method [25].

\section{Statistical analysis}

Mann-Whitney test was used to calculate differences between controls and slices cultured with the tested compounds for ATP, protein, LDH, TG, and bile acids. The cut off for statistical significance was set at a $p$-value $<0.05$.

Mann-Whitney (MW) test was used to determine if there was a significant difference in gene expression assessed by q-PCR in slices exposed to different compounds. A $p$-value $<0.05$ was considered significant.

\section{Results}

Exposure of PCLS to CSA and CPZ

In order to identify non-cytotoxic concentrations of the model cholestatic compounds (CsA and CPZ), dose selection exposure experiments were performed. In these experiments, PCLS were incubated for 24 hours with four different concentrations of each drug. The applied concentrations were for CsA 1, 2, 40, and $100 \mu \mathrm{M}$ and for CPZ 2, 4, 20, and $80 \mu \mathrm{M}$. Viability of the PCLS was assessed by using LDH leakage, ATP, and protein content as parameters. On the basis of the results from the viability measurements, the highest concentration that did not evoke significant decrease in viability was selected for subsequent gene expression profiling experiments. The results from the dose selection experiments are presented in Additional file 1: Figure S1. With respect to CPZ, a concentration of $20 \mu \mathrm{M}$ was chosen for the microarray experiment. Although CsA even at the concentration of $100 \mu \mathrm{M}$ did not induce a decrease in viability, $40 \mu \mathrm{M}$ was selected for the microarray experiment since this concentration was also used in other in vitro studies [26]. Results from the dose selection experiments are presented in Additional file 1: Figure S1A-C. Since the exposure for gene expression profiling was performed with liver slices from mice other than those used for dose selection, the viability of these slices was tested as well. As shown in Figure 1, it could be confirmed that $40 \mu \mathrm{M}$ CsA and $20 \mu \mathrm{M} \mathrm{CPZ}$ did not affect the viability of the slices.

\section{Transcriptome analysis: biological processes and pathway analysis}

Upon treatment of the slices for $24 \mathrm{~h}$ with $40 \mu \mathrm{M}$ CsA or $20 \mu \mathrm{M} \mathrm{CPZ,} \mathrm{RNA} \mathrm{was} \mathrm{isolated} \mathrm{and} \mathrm{hybridized} \mathrm{to}$ DNA microarrays. In order to identify and to compare significantly enriched processes, GSEA was performed using gene sets made in the text-mining tool ANNI. The gene sets (listed in Additional file 4: Table S1) were related to diverse hepatic (e.g. lipid and glucose metabolism, inflammation, bile acid metabolism) and non- hepatic biological processes (e.g. adipogenesis, morphogenesis, tight junctions). As indicated in Figure 2, CsA and CPZ affected several of the gene sets/processes $(p<0.05$, FDR $<0.25)$. Both compounds down-regulated processes related to cholesterol, bile acids, lipid, and glucose metabolism. The gene set related to Kupffer cells was also down-regulated by both compounds. CsA, but not CPZ, up-regulated processes related to inflammation (such as $\mathrm{T}$ cells, immunotoxicity, inflammation, and natural killer cells) and molecular processes involved in cell death (apoptosis, necrosis) and stress response (biological adaptation to stress, and sumoylation). Moreover, CsA downregulated processes related to cirrhosis and stellate cells (Figure 2).

In order to get insight into the molecular mechanisms of action of CsA and CPZ, the significantly altered genes identified by GSEA (1192 and 811 genes for CsA and CPZ respectively) were uploaded into MetaCore to identify significantly altered pathways. In total, we identified 204 


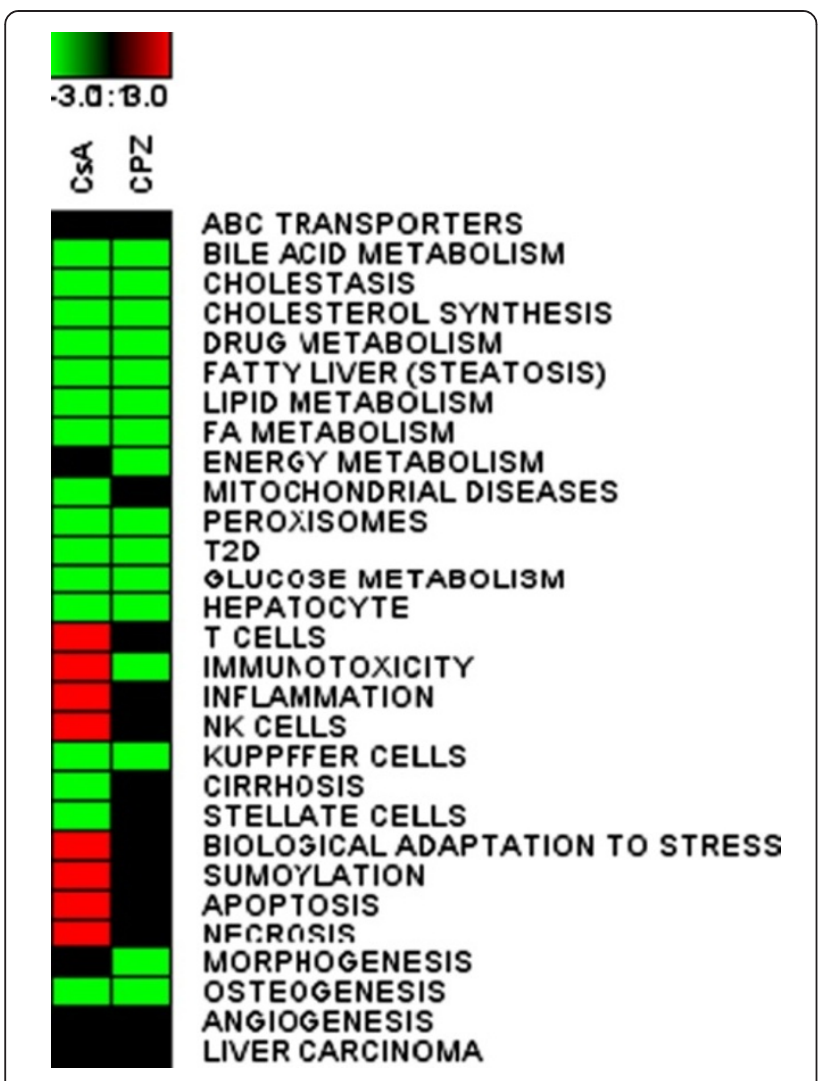

\section{ANNI gene setsp $<0.05$ FDR $<0.25$}

Figure 2 Effects of cholestatic drugs on gene expression in mouse PCLS. PCLS obtained from 5 mice were treated with $40 \mu \mathrm{M}$ cyclosporin A (CsA), $20 \mu \mathrm{M}$ chlorpromazine (CPZ) or vehicle (DMSO) for 24 hours and subjected to Affymetrix microarray analysis. The biological processes in the heat map correspond to gene sets significantly affected according to GSEA analysis ( $p<0.05$, FDR $<$ 0.25). Gene sets were obtained using the ANNI text mining tool. Processes that were up-regulated are represented by red color, the down-regulated processes are depicted in green, processes that were unchanged are depicted in black.

and 125 significantly altered pathways for CsA and CPZ, respectively $(\mathrm{p}<0.005)$ (Additional file 6: Table S3). For a general overview of the processes affected by the drugs, the identified pathways were grouped into broader functional categories using a MetaCore pathway classification output (Table 1, Additional file 6: Table S3). The analysis based on the functional categories showed that there was a substantial overlap between pathways affected by the two drugs (Table 1). Both drugs affected pathways related to immune function (immune response, chemotaxis), energy metabolism (pathogenesis of obesity, regulation of metabolism), and other biological processes (development, apoptosis and survival, signal transduction, cell adhesion, G-protein signalling, protein folding and processing). CsA affected additional functional categories, such as cell cycle and DNA damage (Table 1). In a further analysis, we focused on those pathways that according to MetaCore pathway analysis, were found to be significantly altered either by both drugs or specifically by one drug $(\mathrm{p}<0.005)$. Both CsA and CPZ altered Fxr-regulated cholesterol and bile acids cellular transport $(\mathrm{p}=6.8 \mathrm{E}-07$ and $\mathrm{p}=7.5 \mathrm{E}-08$ respectively), Figure 3A, Additional file 6: Table S3. In this pathway both CsA and CPZ significantly down-regulated Fxr as well as its target genes involved in BA transport such as solute carrier family 10 member 2 (Slc10a2) and multi-drug resistance 1/ ATP-binding cassette, sub-family B, member 1 (Mdr1/Abcb1), Figure 3A. ATP-binding cassette, sub-family $\mathrm{C}$, member $2 /$ multidrug resistance-associated protein 2 (Abcc2/ Mrp2), were down-regulated only by CsA, while Bsep/ ATP-binding cassette, sub-family B, member 11 (Abcb11) and solute carrier family 10 (sodium/bile acid cotransporter family), member $1 / \mathrm{Na}+-$ taurocholate cotransporting polypeptide (Slc10a1/Ntcp) were down-regulated only by CPZ (Figure 3A). Moreover, both CsA and CPZ down-regulated genes coding for cholesterol transporters such as Abcg5/ Abcg8 and Scavenger receptor class B member 1 (Sr-Bi) as well as genes encoding for phospholipids transportermultidrug resistance 3/ ATP-binding cassette, sub-family B (Mdr/Tap), member 4 (Mdr3/ Abcb4/Mdr2), (Figure 3A). Another process significantly affected by both CsA and $\mathrm{CPZ}$ is Bile acids regulation of glucose and lipid metabolism via $\operatorname{Fxr}(\mathrm{p}=7.2 \mathrm{E}-14$ and $\mathrm{p}=1.1 \mathrm{E}-09$ respectively), (Figure 3B). Both, CsA and CPZ led to down-regulation of several genes in this pathway, e.g. Fxr $(\mathrm{Nr} 1 \mathrm{~h} 4)$, retinoid $\mathrm{X}$ receptor $\alpha$ (Rxr $\alpha)$, small heterodimer partner (Shp), hepatocyte nuclear factor 4 alpha $(\mathrm{Hnf} 4 \alpha)$, apolipoprotein B (Apob), very low-density lipoprotein (VLDL/Apoc2), liver $\mathrm{X}$ receptor $\alpha(\operatorname{Lxr} \alpha)$. Furthermore, both CsA and CPZ significantly down-regulated another pathway involved in lipid homeostasis: Rxr-dependent regulation of lipid metabolism via Ppar, Rar and Vdr ( $\mathrm{p}=5.8 \mathrm{E}-05$ and $\mathrm{p}=2.9 \mathrm{E}-04$ respectively), (Figure $3 \mathrm{C}$, Additional file 6 : Table S3).

In addition, CsA and CPZ significantly down-regulated several genes of Cell adhesion_ECM remodelling pathway $(\mathrm{p}=1.3 \mathrm{E}-07$ and $\mathrm{p}=4.1 \mathrm{E}-09$ respectively), (Figure 4, Additional file 6: Table S3). Several metalloproteinases were down-regulated by both drugs (Mmp-12, 15, 16), as well as other genes involved in ECM remodelling (Syndecan-2, Egfr).

One of the most significantly affected pathways by CsA was the Endoplasmic reticulum stress response pathway ( $\mathrm{p}=1.8 \mathrm{E}-13$ ), (Apoptosis and survival), Additional file 6: Table S3, Additional file 7: Figure S4. Several genes present in this pathway were up-regulated, such as NF-kB p50/p65, Grp78, Ire-1, and Traf2. Additionally, CsA affected several pathways related to NF- $\kappa$ B signalling and inflammation, such as $N F-\kappa B$ signaling ( $\mathrm{p}=4.2 \mathrm{E}-11)$, Il-1 signaling pathway $(\mathrm{p}=7.8 \mathrm{E}-11)$, and Hsp60 and Hsp70 Tlr signaling 


\begin{tabular}{lcc}
$\begin{array}{l}\text { Table 1 Overview of pathway functional categories } \\
\text { affected by CsA and CPZ }\end{array}$ & $\begin{array}{l}\text { Nr of pathways } \\
\text { changed by CsA }\end{array}$ & $\begin{array}{c}\text { Nr of pathways } \\
\text { changed by CPZ }\end{array}$ \\
\hline $\begin{array}{lcc}\text { Functional } \\
\text { category }\end{array}$ & 58 & 27 \\
\hline Immune response & 53 & 41 \\
Development & 23 & 16 \\
Pathogenesis of obesity & 18 & 6 \\
Apoptosis and survival & 16 & 12 \\
Signal transduction & 10 & 8 \\
Regulation of metabolism & 5 & 0 \\
Cell cycle & 4 & 8 \\
Cell adhesion & 4 & 2 \\
G-protein signaling & 4 & 2 \\
Protein folding and processing & 3 & 1 \\
Chemotaxis & 3 & 2 \\
Cytoskeleton remodeling & 2 & 0 \\
DNA damage & 1 & 0 \\
Reproduction & &
\end{tabular}

Pathways identified in MetaCore were grouped into functional categories according to the MetaCore classification tool (first column). The number of affected pathways within the functional categories is presented in separate columns for cyclosporin A (CsA) and chlorpromazine (CPZ). MetaCore analysis revealed a total of 204 (CsA) and 125 (CPZ) significantly altered pathways $(p<0.005)$. All identified pathways are presented in Additional file 6: Table S3.

pathway ( $\mathrm{p}=1.7 \mathrm{E}-10)$, (Additional file 6: Table S3, Additional file 8: Figure S8A-C). In general we observed up-regulation of different NF-kB partners (NF-kB p65, p100, p52) and pro-inflammatory cytokines exampled by Tnfo or Il-12.

\section{Identification of biomarkers}

In order to identify biomarkers that could be used for identification of drugs with cholestatic properties, genes were selected that, according to GSEA, significantly contributed to the enrichment of the analysed gene sets $(\mathrm{p}<0.05, \mathrm{FDR}<0.25)$ in both CsA and CPZ treatments. In total 305 common candidate biomarkers were identified and further selection using $\mathrm{FC} \geq 1.5$ as criterion resulted in 73 genes. These genes could be categorized in five functional clusters: $\beta$-oxidation, biotransformation, BA metabolism, BA conjugation, and lipid metabolism (Figure 5). Hierarchical clustering of these genes, led to a very good separation between control and slices treated with CsA (Figure 6A). CPZ treated samples separated very well except for one control and one treated sample, which were mis-classified (Figure 6B). Furthermore, we analysed the expression levels of these 73 genes in liver slices exposed to two other classes of hepatotoxicants, i.e. steatogenic drugs (valproic acid and amiodarone) and necrotic drugs (paraquat and isoniazid). In contrast to the slices exposed to CsA and CPZ, hierarchical clustering of array data (of the 73 selected genes) from slices exposed to steatogenic (Figure 6C-D) and necrotic compounds (Figure 6E-F) did not show a clear separation between treatment and control.

\section{Microarray data verification ( $q P C R$ )}

For verification of the array data, 4 genes out of the 73 potential biomarkers were subjected to qPCR. These genes were Baat, Abcg5, Abcg8, and Klf15. The major criterion for the selection of these genes was related to their function in Fxr signalling. Three of these four genes are related to BA metabolism and are targets of Fxr/Nr1h4 [2]. Moreover, mutations in Baat, Abcg5 or Abcg8 have been shown to be involved in the development of cholestasis in humans [2,8]. Although Klf15 is not a Fxr target, it is known to be involved in gluconeogenesis [27], which is regulated by Fxr [28,29]. More detailed information about the functions of the selected genes is presented in Additional file 9: Table S4. The qPCR experiments were performed on the same RNAs as used for the DNA microarray hybridisations. Statistical analysis of the qPCR data showed that CsA and CPZ significantly affected 3 out of the 4 candidate biomarkers (Table 2A-B). In slices exposed to necrotic and steatogenic drugs the expression of at most one out of the four genes was significantly affected (Table 2C-F).

\section{Biochemical and histological analysis}

Slices cultured for 24 and 48 hours with the reference compounds and controls were subjected to histological analysis. Upon incubation for 24 hours, some hepatocytes localized in the outer part of slices treated with CsA had developed a characteristic ballooned phenotype, which was even more evident after 48 hours and was absent in control slices. These hepatocytes were enlarged compared to control and had their nucleus in the centre (Figure 7). Based on the histological examination as well as transcriptome data analysis, showing alternations in lipid and BA metabolism, it could be envisaged that the enlargement of cells treated with CsA is due to TG or BA accumulation. Therefore, biochemical assays to quantify TG and BA levels were employed. However, compared to control, no increase of BA or TG in CsA treated slices was observed (Figure 8A and B). Additionally, we performed Periodic acid-Schiff (PAS) staining to detect glycogen, which theoretically could also accumulate in hepatocytes and lead to their enlargement. As expected, control slices showed substantial number of cells containing glycogen (Figure $8 \mathrm{C}$ ). However, in the enlarged cells of the CsA treated slices no glycogen could be detected (Figure 8D). Finally, Fouchet staining was performed to examine whether CsA treatment resulted in the accumulation of bile, a process that is often accompanying cholestasis. Although, we did not detect bile accumulation in slices, Fouchet staining unexpectedly 


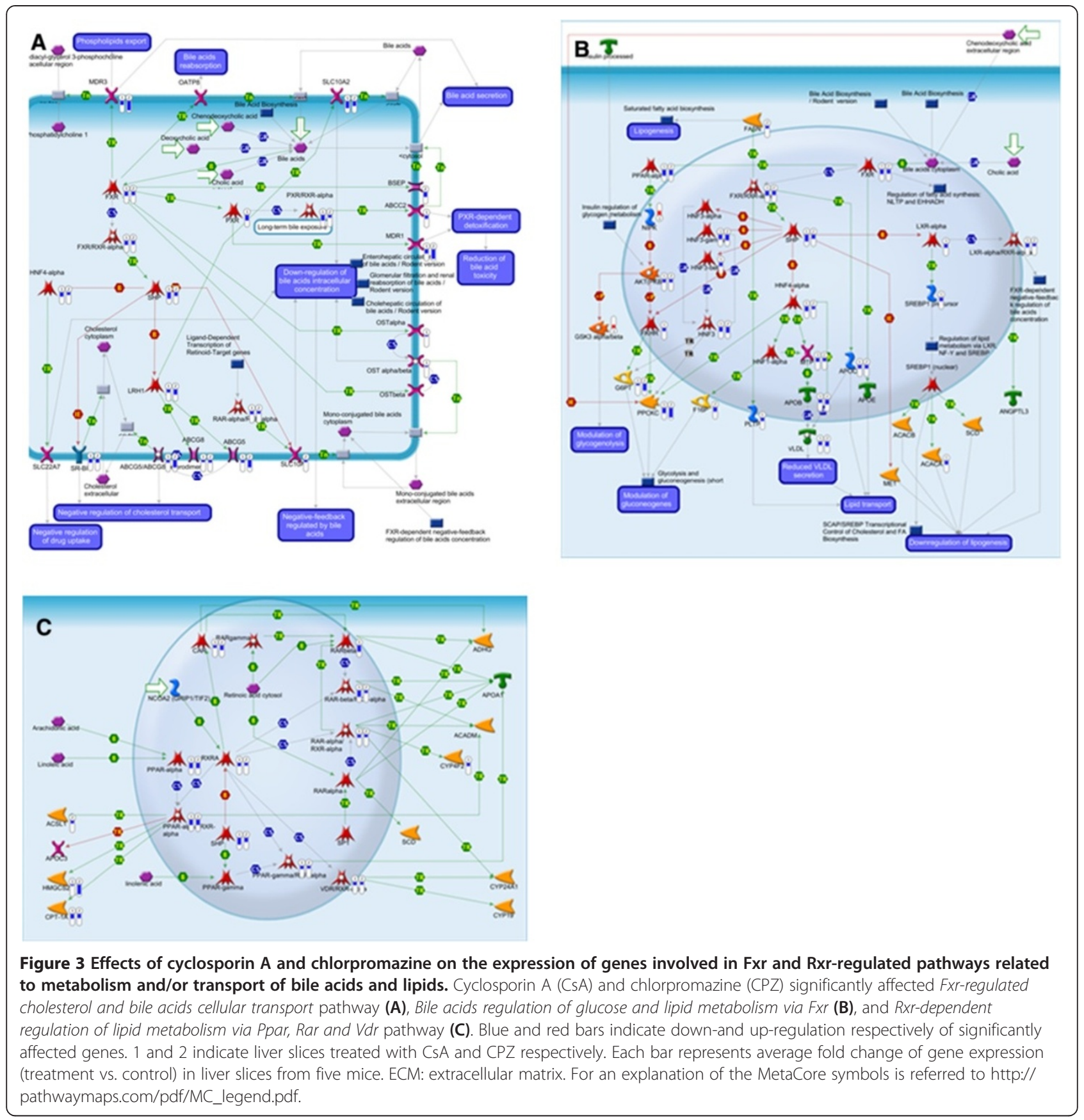

showed the presence of several vacuoles, which were absent in control slices (Figure 8E and F).

\section{Comparative data analysis: relevance for human pathology}

In order to verify whether the results on drug induced cholestasis in mouse liver slices would also be observed in patients suffering from cholestasis, publically available transcriptomics data were analysed. The data were generated using liver biopsies obtained from children suffering from biliary atresia, intrahepatic cholestasis of other origin than biliary atresia, and age-matched controls. GSEA revealed that patients suffering from both biliary atresia and intrahepatic cholestasis had significantly down-regulated gene sets related to different aspects of energy metabolism including bile acid metabolism, FA metabolism or peroxisomes. These changes were in line with drug-induced cholestasis in mouse PCLS (Figure 9). However, gene sets related to ECM, angiogenesis, and fibrosis were up-regulated in the livers of cholestatic patients but 


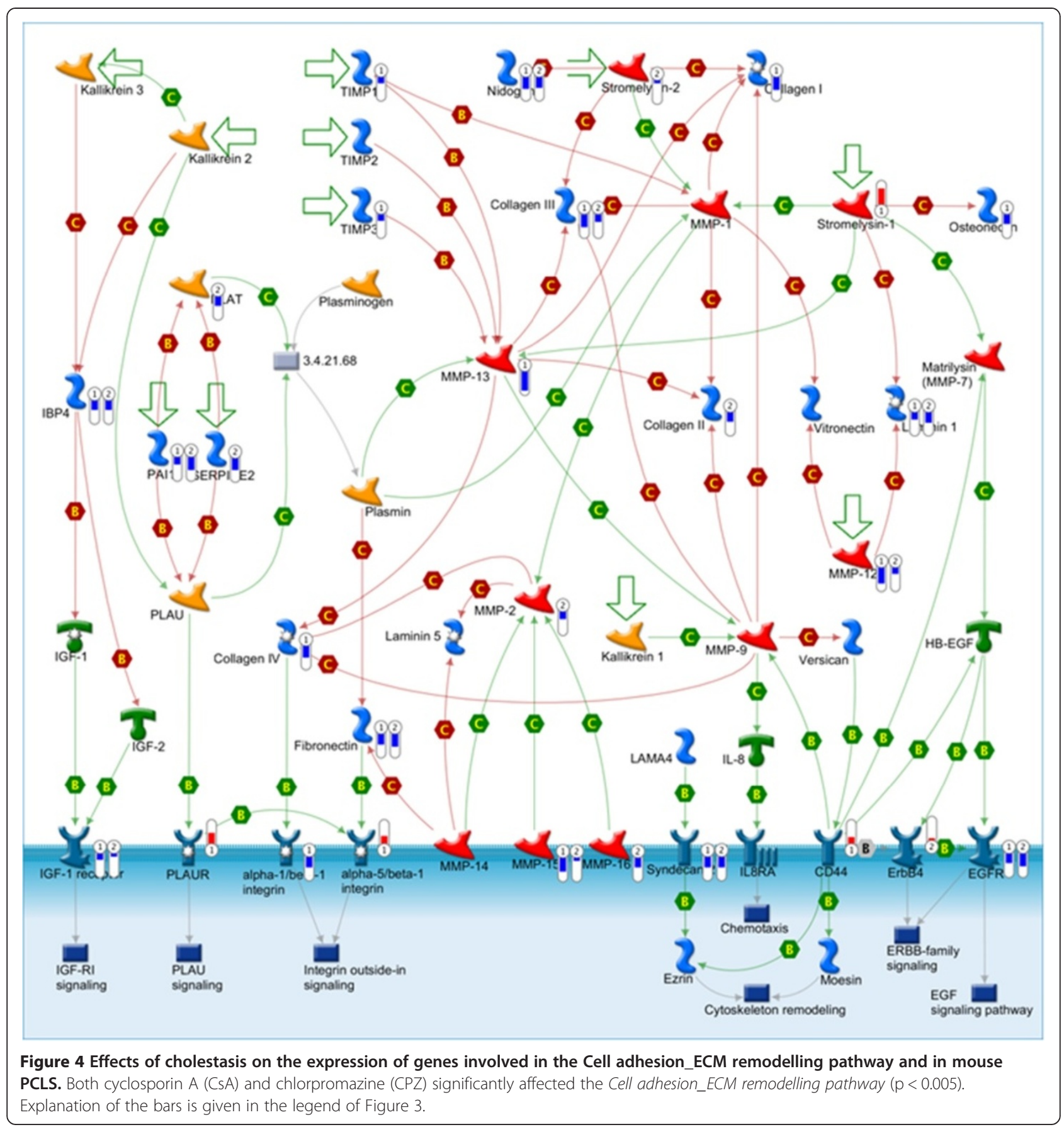

the same processes were either not affected or altered in the opposite direction by the tested drugs in mouse liver slices (Figure 9). MetaCore pathway analysis showed that FXR signalling such as Bile acids regulation of glucose and lipid metabolism via FXR (Additional file 10: Figure S6A) and FXR-dependent negative-feedback regulation of bile acids concentration (Additional file 10: Figure S6B) were significantly down-regulated in cholestatic patients. These latter two pathways were also found to be down-regulated in the mouse slices experiment (Figure 3). Furthermore, not only GSEA but also MetaCore analysis showed that the pathway ECM remodeling was up-regulated (Additional file 11: Figure S7). This pathway was downregulated by CsA -and CPZ-treatment in mouse PCLS (Figure 4).

\section{Discussion}

In the present study, we aimed to assess whether mouse PCLS can be used as an ex vivo model to study processes related to the development of drug-induced cholestasis. 


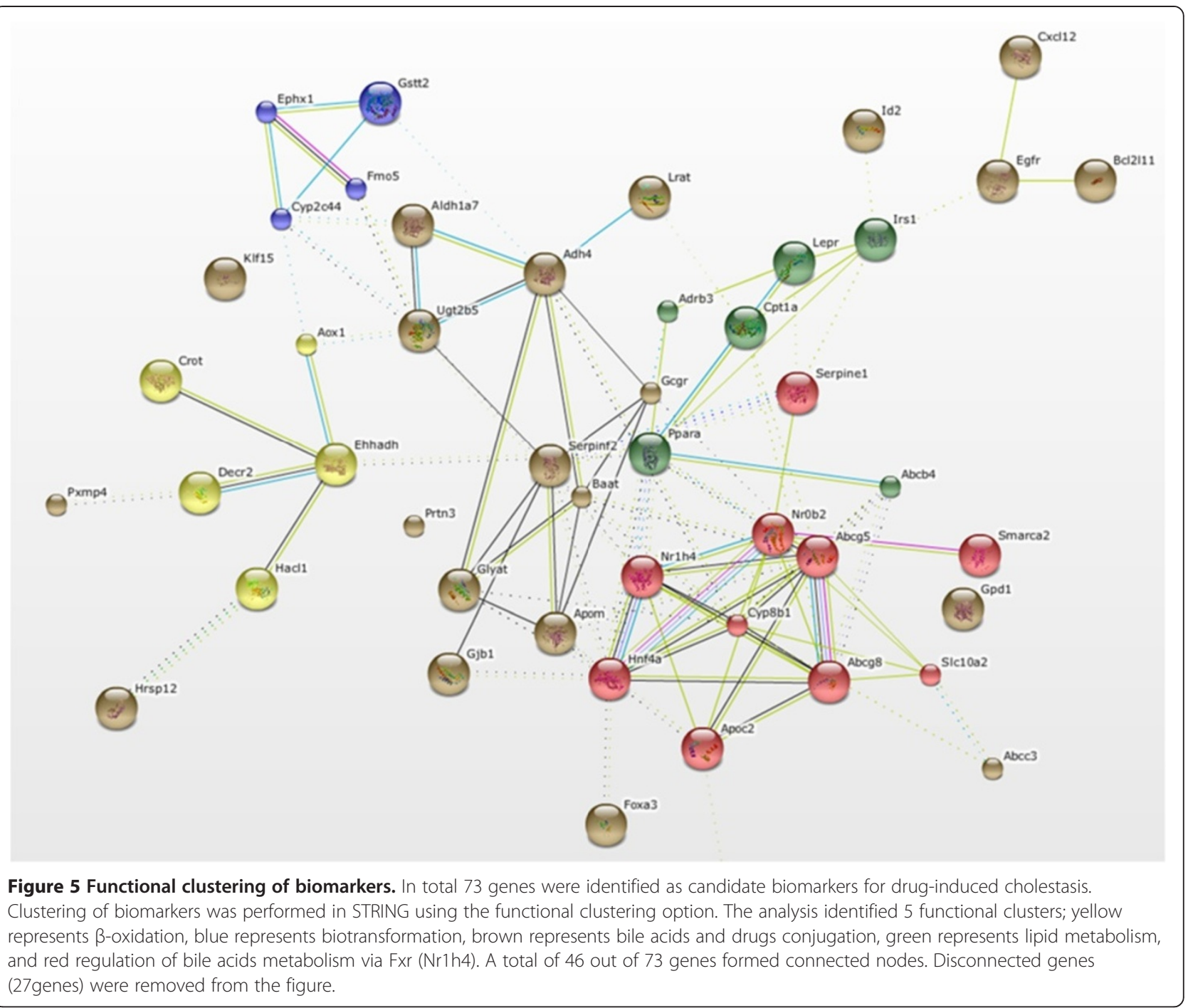

$\mathrm{CsA}$ and $\mathrm{CPZ}$ were used as reference compounds known to induce cholestasis in vivo in humans and rodents. In depth transcriptome analysis combined with biochemical and histological evaluation were performed to identify early molecular events and biomarkers that can be used to screen drugs for cholestatic properties.

\section{Exposure of PCLS to CsA and CPZ}

The effects of CsA and CPZ were analysed upon treatment of PCLS with $40 \mu \mathrm{M}$ CsA and $20 \mu \mathrm{M} \mathrm{CPZ}$. These concentrations were relatively high compared to plasma levels of patients chronically treated with the same drugs, i.e. 0.04-0.1 $\mu \mathrm{M}$ for CsA [26] and 0.02-0.3 $\mu \mathrm{M}$ for CPZ [30], but similar concentrations were used in other in vitro studies $[30,31]$ and the applied concentrations did not cause a decrease in slices viability assessed ATP and LDH assays.
However, GSEA analysis on the transcriptome data revealed that CsA induced a higher number of processes indicative for cytotoxicity such as apoptosis, necrosis, or inflammation than CPZ (Figure 2). This could indicate that the applied concentration of CsA was toxic, although according to the viability tests (ATP and LDH assays) doses even higher than $40 \mathrm{uM}$ did not cause a significant decrease in viability (Additional file 1: Figure S1). These results suggest therefore, that ATP as well as LDH measurements may not always be reliable to predict cytotoxic concentration of drugs. In addition, due to known interspecies differences for toxic doses of drugs, application of drug concentrations identical to in vitro studies using different species, seems to be inappropriate as well $[32,33]$. On the other hand, we cannot exclude that the cytotoxic processes are due to pharmacological actions of CsA, because other in vitro studies demonstrated that also lower CsA concentrations induce 


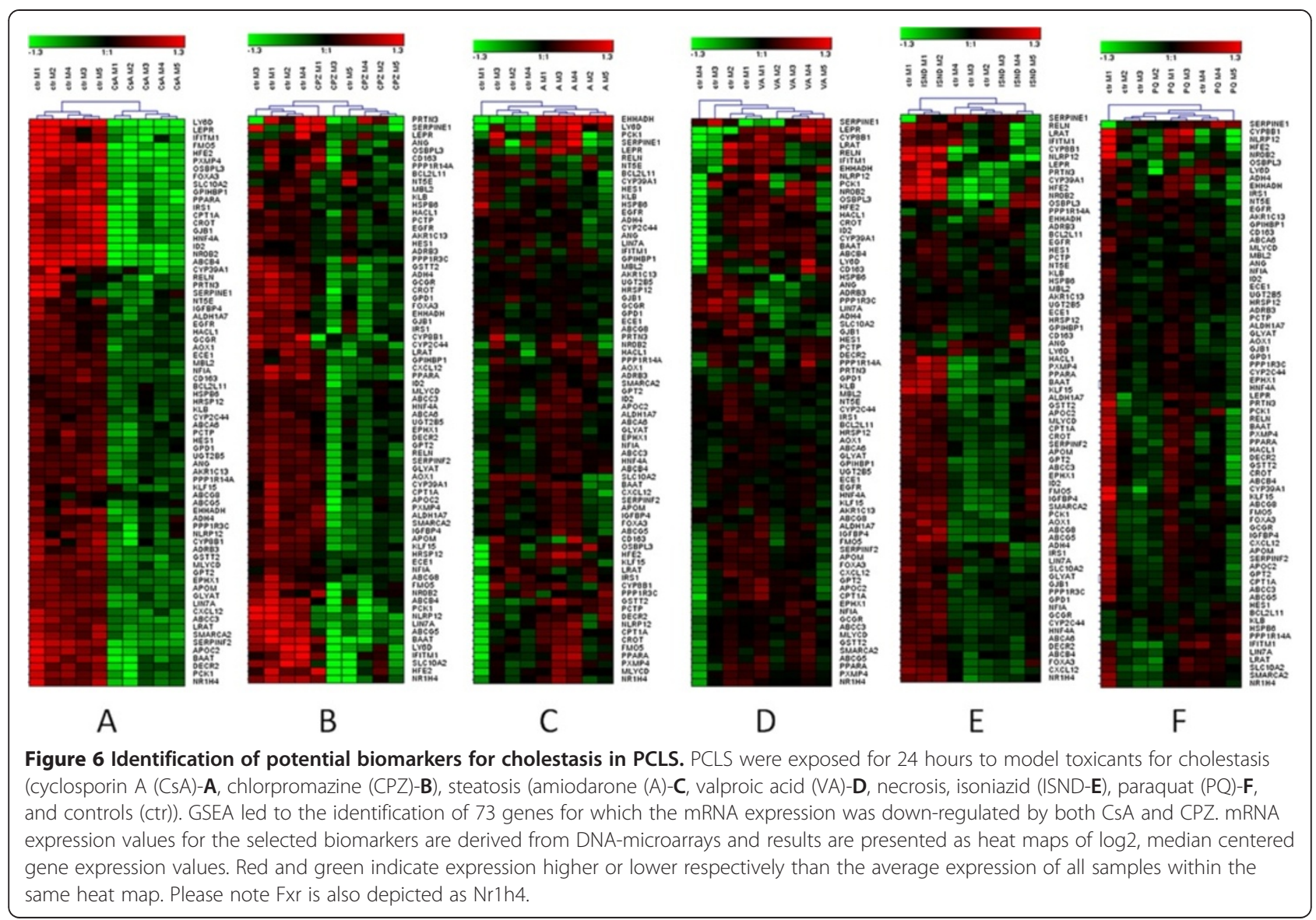

toxicity-related processes such as ER stress, apoptosis, and cystic fibrosis [26].

Subsequent detailed pathway analysis revealed that both CsA and CPZ significantly altered pathways $(\mathrm{p}<0.005)$ governed by Fxr including Fxr regulated cholesterol and BAs cellular transport (Figure 3A, Additional file 6: Table S3) and $B A$ regulation of glucose and lipid metabolism via Fxr (Figure 3B, Additional file 6: Table S3). Both drugs downregulated several transporters involved in bile production and BA secretion: Mdr1, Abcg5/Abcg8, as well as Mdr3 (Mdr2). This is in agreement with a previous rat PCLS study, which showed that CsA reduced Mdr1 activity [30]. Furthermore, we observed that CsA down-regulated another BA transporter, Mrp2 (Abcc2). These data are in line with observations made in rats showing that the same transporters were down-regulated by CsA in the liver at the protein level [34]. With regard to CPZ we detected down-regulation of Fxr targets such as Bsep, Mdr3, Ntcp (Slc10a1), and Cyp8b1. These findings are in agreement with a recent study showing a down-regulation of the same genes in CPZ-treated HepaRG cells [35]. The importance of Fxr signalling in the maintenance of BA homeostasis in vivo is unequivocal and it was demonstrated that Fxr knockout mice have disturbed BA homeostasis
[36]. Moreover, we observed that both CsA and CPZ down-regulated several genes related to lipid metabolism

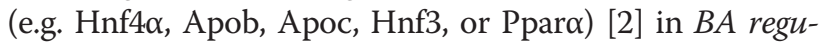
lation of glucose and lipid metabolism via Fxr pathway (Figure 3B, Additional file 6: Table S3). Consistent with these observations, it was reported that CsA treatment led to an increase in accumulation of lipid droplets in rat primary hepatocytes after 24 hours [37] and CsA therapy has been implicated in the development of fatty liver [38]. Next to that, CPZ has been shown to inhibit $\beta$-oxidation in vivo [39] and to evoke an increase in microsomal phospholipidosis in rats [40]. However, based on biochemical measurement of TG in our study, we concluded that no lipids accumulated in CsA or CPZ treated slices. Based on the outcome of the present work, we propose that CsA and CPZ down-regulate Fxr signalling resulting in perturbation of BA, glucose, and lipid metabolism, which eventually leads to the development of both cholestasis and metabolic disorders. This is in line with the known functions of Fxr related to the regulation of both BA and energy homeostasis $[10,36]$ and common side effects of CsA and CPZ treatments, i.e.cholestasis, fatty liver, and type 2 diabetes [38,40]. Additionally, we detected that both CsA and CPZ down-regulated other NRs involved in 
Table 2 Validation of potential biomarkers for cholestasis in PCLS

\begin{tabular}{|c|c|c|c|c|c|c|c|c|c|c|c|c|c|}
\hline \multicolumn{7}{|l|}{ A } & \multicolumn{7}{|l|}{ B } \\
\hline Drug & CsA & CsA & CsA & CsA & CsA & MW test & Drug & $\mathrm{CPZ}$ & $C P Z$ & $C P Z$ & $C P Z$ & $\mathrm{CPZ}$ & MW test \\
\hline Gene & $\mathrm{FC} 1$ & FC2 & FC3 & FC4 & FC5 & & Gene & FC1 & FC2 & FC3 & FC4 & FC5 & \\
\hline ABCG5 & -3.5 & -1.8 & -1.0 & -1.3 & -1.2 & NS & ABCG5 & -4.2 & -2.9 & -6.7 & -3.8 & 1.0 & * \\
\hline ABCG8 & -4.9 & -3.9 & -1.2 & -2.0 & -1.2 & $*$ & ABCG8 & -2.9 & -2.1 & -5.4 & -4.5 & -1.1 & * \\
\hline BAAT & -5.2 & -4.2 & -1.6 & -2.2 & -1.7 & * & BAAT & -2.3 & -3.3 & -8.5 & -6.4 & 1.4 & * \\
\hline KLF15 & -1.2 & -1.7 & -2.0 & -1.4 & -1.3 & * & KLF15 & -2.0 & -1.8 & -3.2 & -1.8 & 1.6 & * \\
\hline \multicolumn{7}{|l|}{$C$} & \multicolumn{7}{|l|}{ D } \\
\hline Drug & A & A & A & $A$ & A & MW test & Drug & VA & VA & VA & VA & VA & MW test \\
\hline Gene & $\mathrm{FC1}$ & $\mathrm{FC} 2$ & FC3 & FC4 & FC5 & & Gene & FC1 & $\mathrm{FC2}$ & FC3 & FC4 & FC5 & \\
\hline ABCG5 & 1.2 & -1.6 & 1.2 & 1.1 & -1.3 & NS & ABCG5 & 2.0 & -1.7 & -1.1 & 1.4 & 1.6 & NS \\
\hline ABCG8 & 2.0 & -1.6 & 1.1 & 1.1 & -1.1 & NS & ABCG8 & 2.2 & -1.5 & -1.3 & 1.4 & 1.9 & NS \\
\hline BAAT & 1.8 & -2.2 & 1.1 & -1.0 & -1.7 & NS & BAAT & 1.3 & 1.0 & -2.0 & 1.4 & 2.5 & NS \\
\hline KLF15 & 2.6 & -1.8 & -1.5 & -1.4 & -1.3 & NS & KLF15 & 1.0 & -1.9 & -1.7 & -1.1 & 1.1 & NS \\
\hline \multicolumn{7}{|l|}{$E$} & \multicolumn{7}{|l|}{$\mathrm{F}$} \\
\hline Drug & ISND & ISND & ISND & ISND & ISND & MW test & Drug & $P Q$ & $P Q$ & $P Q$ & $P Q$ & $P Q$ & MW test \\
\hline Gene & $\mathrm{FC} 1$ & FC2 & FC3 & FC4 & FC5 & & Gene & $\mathrm{FC} 1$ & FC2 & FC3 & FC4 & FC5 & \\
\hline ABCG5 & 2.0 & 3.0 & 1.1 & 2.0 & 2.0 & * & ABCG5 & 1.1 & 1.1 & 1.7 & -1.3 & -1.6 & NS \\
\hline ABCG8 & 2.1 & 2.8 & 1.0 & 2.3 & 1.9 & NS & ABCG8 & 1.0 & -1.2 & 1.8 & -1.3 & -1.4 & NS \\
\hline BAAT & 1.8 & 1.7 & 1.1 & -1.3 & 1.3 & NS & BAAT & -1.2 & -1.0 & 1.7 & 1.1 & -1.4 & NS \\
\hline KLF15 & 1.2 & 2.4 & 1.0 & 1.1 & 1.2 & NS & KLF15 & 1.3 & -1.0 & 1.3 & 1.1 & -1.2 & NS \\
\hline
\end{tabular}

PCLS were exposed for 24 hours to model toxicants for cholestasis (cyclosporin A (CsA)-A, chlorpromazine (CPZ)-B), necrosis (isoniazid (ISND)-C, paraquat (PQ)-D), and steatosis (amiodarone (A)-E, valproic acid (VA)-F). Q-PCR was used to measure mRNA expression relative to actin $\beta$ of four potential biomarkers: Abcg5, Abcg8, Baat, and Klf15. FC stands for fold change (treatment vs. control). Q-PCR was performed for liver slices obtained from five biological replicates. Asterisk (*) indicates significantly different gene expression $(P<0.05)$ from controls according to Mann-Whitney-test (MW). NS stands for not significant.

detoxification of BA and lipid metabolism, such as Car (Nr1i3), Vdr and other genes related to lipid metabolism such as Cptla and Hmgcs2 [9], (depicted in Rxr-dependent regulation of lipid metabolism via Ppar, Rar and Vdr pathway, Figure 3C, Additional file 6: Table S3). Down- regulation of these genes possibly adds to the cholestatic and lipogenic properties of the analysed drugs.

Furthermore, for both CsA and CPZ, significant alternations in ECM remodelling pathway were found (Figure 4). It was suggested before, that changes related to ECM

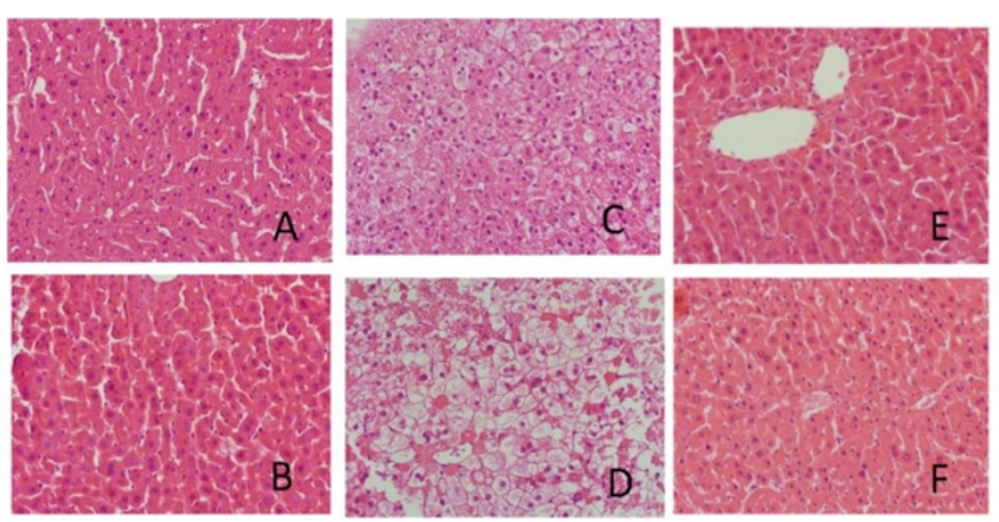

Figure 7 Histological analysis of liver slices treated with CsA and CPZ. PCLS were cultured for 24 and 48 hours in the presence of DMSO (control) (A and B), $40 \mu \mathrm{M}$ CsA (C and $\mathbf{D})$ or $20 \mu \mathrm{M}$ CPZ (E and $\mathbf{F})$. Histology of PCLS treated with CsA revealed ballooning of hepatocytes at the outer parts of slices after 24 and 48 hours (C and $\mathbf{D}$ respectively). Histology of CPZ treated slices cultured for 24 and 48 hours $(\mathbf{E}$ and $\mathbf{F}$ respectively) revealed a slight increase in number of cells containing pycnotic nuclei compared to control slices cultured equally long (A and $\mathbf{B})$. 

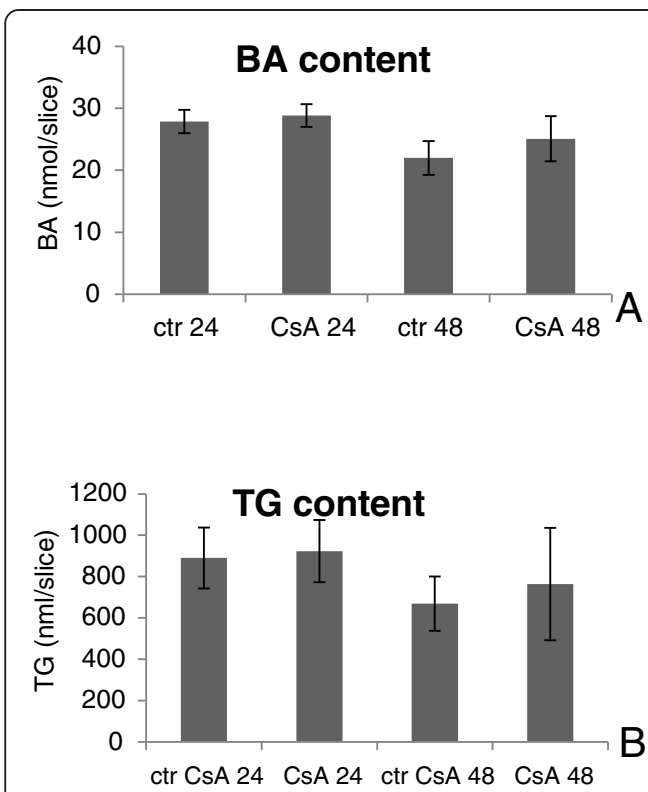
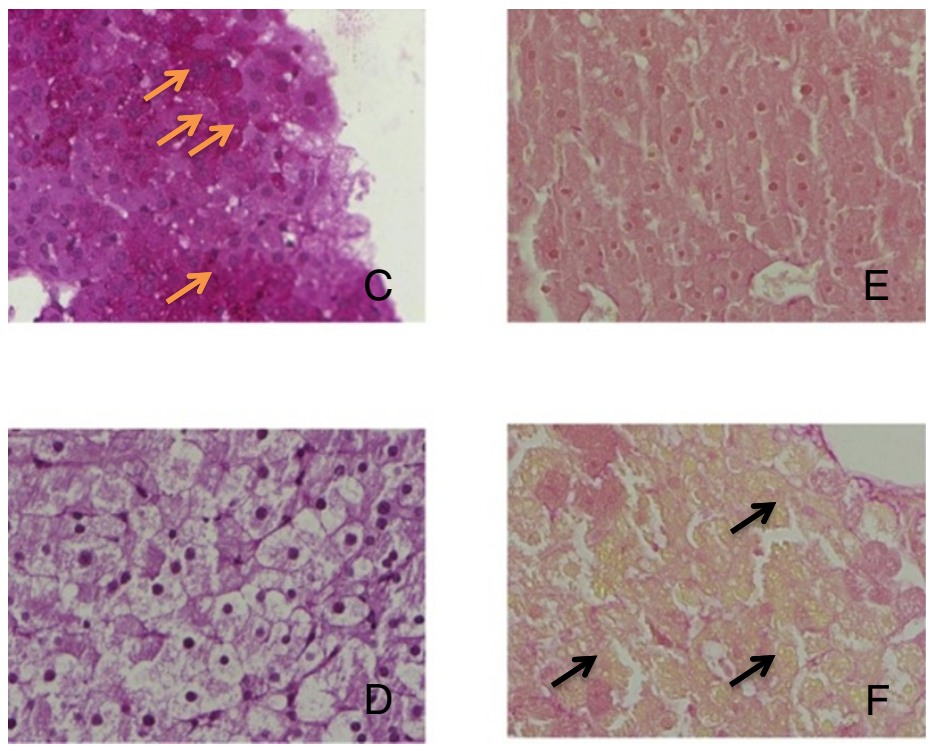

Figure 8 Biochemical analysis and specific histological staining of liver slices treated with CsA. Bile acid (BA) (A) and triglyceride (TG) (B) levels in PCLS cultured for 24 or 48 hours in the presence of either DMSO or $40 \mu \mathrm{M}$ cyclosporin A (CsA) were not affected by any of the conditions, $\mathbf{X}$ - axis represents the tested conditions and Y-axis represents content of BA (A) or TG (B) in nmol per slice. Glycogen was assessed by PAS staining in PCLS cultured for $24 \mathrm{~h}$ in the presence of DMSO (C) or $40 \mu \mathrm{M}$ CSA (D) demonstrating the absence of glycogen in ballooning hepatocytes. Arrows in (C) indicate the presence of glycogen in controls. Fouchet staining in PCLS cultured for 24 h in the presence of DMSO (E) or $40 \mu \mathrm{M}$ CsA (F) demonstrating accumulation of several vacuoles in CsA treated slices (see arrows in F). All pictures were taken using 100-fold magnification.

remodelling could lead to perturbations in intracellular signalling affecting Fxr related pathways and thereby leading to liver injuries [41].

Transcriptome data analysis also revealed processes and pathways that were specifically affected by either CsA or CPZ. CsA treatment up-regulated pathways and genes related to endoplasmic reticulum (ER) stress (Additional file 7: Figure S4), NF- $\mathrm{kB}$ signalling, and inflammation with upregulated cytokines such as Tnf $\alpha, \mathrm{Crp}$, and Il-1 $\beta$ (Additional file 8: Figure S5A-C). These findings are in line with previous reports describing ER stress and inflammation as the primary events in CsA induced toxicity $[42,43]$. ER stress is known to activate NF-kB, which is a key regulator of diverse immune responses [44]. Moreover, we found that CsA up-regulated gene sets related to $\mathrm{T}$ cells. This contrasts to findings that CsA inhibits T-cell receptor signalling and induces Tgf $\beta$, which both prevent the production of cytokines and block the immune system [42]. Inasmuch as CsA caused up-regulation of gene sets related to functions of $\mathrm{T}$ cells, the immunosuppressive effects of CsA in our model, seem to be different compared to CsA effects in vivo. A possible cause could be the relatively short time of this study or a lack of blood immune cells which interactions with the liver immune cells are necessary to fully develop the immune response [45]. To the best of our knowledge, there is only one report describing presence and actions of $\mathrm{T}$ cells in human liver slices [46]. Therefore, the exact biological meaning of the CsA effect on gene expression specific to T-cells in mouse PCLS remains to be elucidated in the future. Additionally, CsA down-regulated the expression of several genes encoding heat shock proteins (HSP) (e.g. Hspb6, Hsph1, Hspe1) as well as Sec proteins (Sec61a1, Sec14l2, Sec1414) (shown in Additional file 12). HSP proteins and Sec proteins are involved in folding/ unfolding of proteins and protein translocation, respectively [47,48]. It is known that ER stress induced by CsA negatively affects the function of the HSP and Sec proteins that eventually lead to perturbations in protein and bile secretion $[49,50]$. Also the vacuolisation in CsA treated slices, as shown in the present study by Fouchet staining, could be a consequence of this disturbance in protein folding and translocation/ secretion $[26,49,51]$.

The up-regulation of ER stress, NF- $\mathrm{kB}$ signalling and expression of pro-inflammatory cytokines (e.g. Tnfo, Crp, Il1 $\beta$ ) by CsA could be the cause of the observed downregulation of Fxr and its gene targets as well as other nuclear receptors. Previously it has been shown that the NF- $\mathrm{kB}$-mediated acute phase response induced by lipopolysaccharide (LPS) is associated with a decrease of Fxr expression in mouse liver [52]. Furthermore, TNF $\alpha$ and IL1 $\beta$ treatment of Hep3B human hepatoma cells and mice resulted in decreased expression of RXR $\alpha$, PPAR $\alpha$, PPAR $\gamma, \mathrm{LXR} \alpha$, as well as their co-activators PGC1 $\alpha$ and 


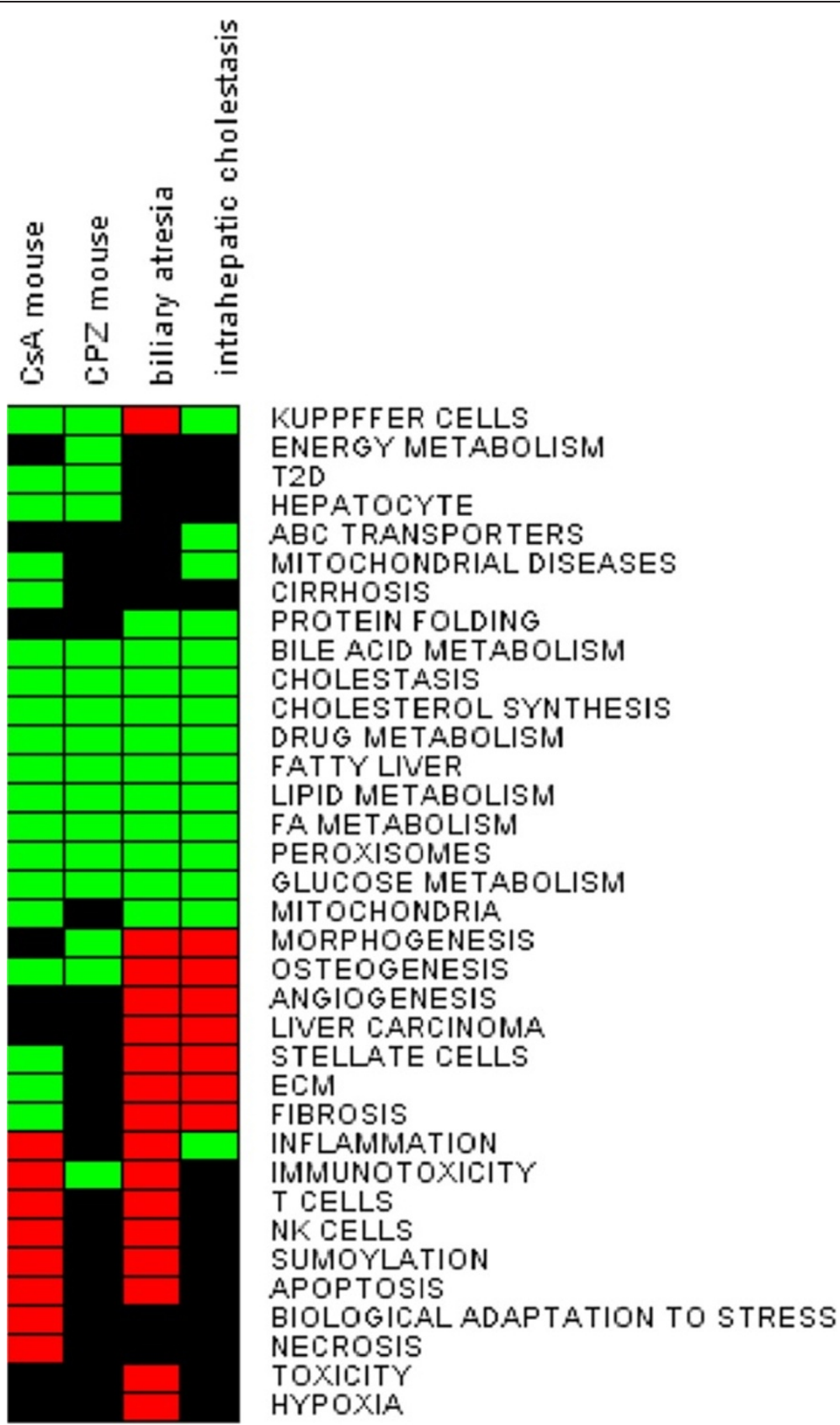

Figure 9 Effects of cholestasis on gene expression in human liver biopsies. A publically available transcriptomics data set (GSE46960) of liver biopsies obtained from 64 infants with biliary atresia, 14 infants with intrahepatic cholestasis of other origin than biliary atresia, and 7 agematched controls were subjected to GSEA. The biological processes in the heat map correspond to gene sets significantly affected according to GSEA analysis $(p<0.05, F D R<0.25)$. Gene sets were obtained using the ANNI text mining tool. Processes that were up-regulated are represented by red color, the down-regulated processes are depicted in green, and processes that were unchanged are depicted in black.

PGC-1 $\beta$, which may contribute to the cytokine induced modulations in hepatic energy homeostasis [53].

Although CPZ did not induce oxidative stress based on transcritpome data, it is likely that it is also a trigger to down-regulate Fxr signaling at early time points that we did not test. Consistent with this notion, it was shown that CPZ induces oxidative stress in HepaRG cells already after 30 min resulting in down-regulation of FXR-target genes after 24 hours [35].

Moreover, we observed that $\mathrm{CPZ}$ and CsA downregulated gene sets related to Kupffer cells (Figure 2) pointing out towards known immunosuppressive actions of CPZ and CsA [54]. Analysis of the microarray data to identify potential biomarkers for cholestasis showed a 
substantial number of genes separating the two cholestatic compounds from non-cholestatic hepatotoxicants (Figure 6A-F). Functional clustering of these potential biomarkers identified processes such as $\beta$-oxidation, biotransformation, BA metabolism and conjugation, and lipid metabolism. To verify the gene expression data obtained with microarrays, q-PCR was performed on genes involved in processes governed by Fxr i.e. BA homeostasis and gluconeogenesis $[2,27,29]$. The expression of the selected biomarkers as assessed by q-PCR was similar to that of the microarray analysis (Tables $2 \mathrm{~A}-\mathrm{F}$ ). To further validate the potential of these genes (Figure 6) to screen for cholestasis additional studies are required using more reference compounds and models.

\section{Biochemical and histological analysis}

Based on the transcriptome data, it was expected that TG or BA levels would be altered in PCLS upon the treatment with CsA and CPZ. However, this could not be confirmed by biochemical and histological assays. Fouchet staining, reacting with bilirubin is not suitable to identify bile accumulation ex vivo probably due to lack of bilirubin derived from broken heme that in vivo reaches the liver via the blood. On the other hand, H\&E staining demonstrated that CsA, but not CPZ, induced ballooning of hepatocytes. This phenotype is generally regarded as a form of apoptosis in the context of inflamed fatty liver (steatohepatitis) caused by obesity, alcohol or other toxic compounds $[55,56]$. This phenotype could be associated with the up-regulation of gene sets related to apoptosis and inflammation observed in CsA treated slices.

It has to be underlined that the mouse PCLS experiments were performed without supplementing the media with BA. On the one hand this might explain the absence of BA accumulation in the slices upon treatment with CsA and CPZ. On the other hand, by leaving out BA from the culture medium, we have identified early events involved in the development of CsA- and CPZ-induced cholestasis rather than indirect effects associated with a late cholestatic phenotype, such as toxic BA accumulation.

\section{Relevance of the mouse PCLS findings for human}

Due to the lack of transcriptomic data of cholestatic livers from patients treated with CsA and CPZ, we used publically available data of livers of patients suffering from cholestasis due to biliary atresia and other causes. The comparison of the gene expression profiles of druginduced cholestasis in mouse PCLS and livers of cholestatic patients pointed to several processes that were similarly affected. These processes are associated with energy metabolism and include lipid, BA metabolism, and glucose metabolism, among others (Figure 9, Additional file 10: Figure S6). The comparative analysis thereby indicates that mouse PCLS are a valid model to study mechanisms involved in the development of human cholestasis. The opposite direction in regulation of gene sets and pathways related to ECM, angiogenesis and fibrosis between livers of cholestatic patients and mouse PCLS (Figure 9, Additional file 11: Figure $\mathrm{S} 7$ ) could be explained by obvious differences related to e.g. chronic pathological conditions in vivo vs. short time experiments in PCLS. The same is true for up-regulation, only in patients, of other gene sets related to liver carcinoma, angiogenesis or fibrosis (Figure 9) that, in order to develop, require long-term processes as well as multi-organ and blood cells interactions [2,57]. Consistent with these notions, it was also reported that cholestasis and other chronic liver diseases are associated with the development of fibrosis caused by accumulation of ECM [58].

However, whether oxidative stress and subsequent downregulation of NRs involved in the maintenance of BA and energy homeostasis are the common links between drug-related cholestasis in mouse (PCLS) and drugunrelated cholestasis in humans, remains to be resolved in the future, when more appropriate human data will be available.

\section{Conclusion}

In summary, our study demonstrates that mouse PCLS can be used as a tool to identify mechanisms of action of cholestatic compounds. Based on the transcriptome analysis it is proposed that CsA and CPZ affect pathways involved in general stress responses, such as ER stress (CsA), NF-kB-mediated responses (CsA), and ECM remodelling (CsA and $\mathrm{CPZ}$ ) associated with down-regulation of Fxr signalling, a key process in BA and lipid homeostasis. Importantly, gene expression pattern in livers of cholestatic patients displayed several similarities to PCLS treated with model cholestatic compounds, indicating that some common mechanisms are involved in the development of cholestasis both in human pathology and mouse PCLS. Moreover, this work provides a set of genes that are potentially useful to screen compounds for Fxr-mediated cholestatic properties.

\section{Additional files}

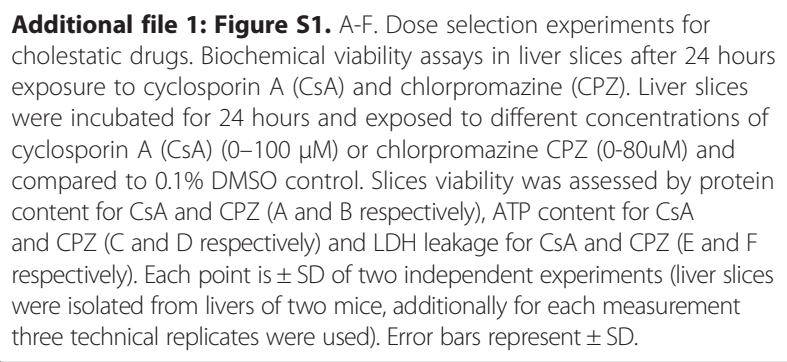


Additional file 2: Figure S2. A-C. Dose selection experiments for steatogenic drugs. Biochemical viability assays in liver slices after 24 hours exposure to amiodarone (A) and valproic acid (VA). Liver slices were incubated for 24 hours and exposed to different concentrations of $A$ (0-100 $\mu \mathrm{M})$ or VA (0-500 $\mu \mathrm{M})$ and compared to corresponding controls. Slices viability was assessed by protein content, ATP content and LDH leakage. Each point is \pm SD of five independent experiments (liver slices were isolated from livers of five mice, additionally for each measurement two technical replicates were used).

Additional file 3: Figure S3. A-C. Dose selection experiments for necrotic drugs. Biochemical viability assays in liver slices after 24 hours exposure to isoniazyd (ISND) and paraquat (PQ). Liver slices were incubated for 24 hours and exposed to different concentrations of ISND (0-1000 $\mu \mathrm{M})$ or PQ $(0-10 \mu \mathrm{M})$ and compared to corresponding controls. Slices viability was assessed by protein content, ATP content, and LDH leakage. Each point is \pm SD of five independent experiments (liver slices were isolated from livers of five mice, additionally for each measurement two technical replicates were used).

Additional file 4: Table S1. ANNI gene sets.

Additional file 5: Table S2. List of primers used for q-PCR.

Additional file 6: Table S3 Total pathway analysis. Pathway analysis led to identification of 204 and 125 significantly altered pathways for CsA $(A)$ and $C P Z(B)(p<0.005)$. Pathways were grouped into functional categories according to MetaCore pathways classification (first column). Each functional category contains pathway name and $p$ value. Additionally number of significantly affected genes and total number of gene in pathways are given. Pathways that were discussed in the paper in details are in bold and underlined.

Additional file 7: Figure S4. MetaCore pathway analysis in CSA treated slices: Endoplasmic reticulum stress response pathway. Cyclosporin A (CSA) significantly affected Endoplasmic reticulum stress response pathway $(p<0.005)$. Blue (down-regulation) and red (up-regulation) bars indicate significantly affected genes. The numbers $1-5$ represent fold change (treatment vs. control) of gene expression of five independent experiments (i.e. liver slices isolated from 5 mice).

Additional file 8: Figure S5. MetaCore pathway analysis in CsA treated slices: Nf-KB signaling II-1 signaling pathway, and Hsp60 and Hsp70 T/r signaling pathways. PCLS treated with CSA displayed significant $(\mathrm{p}<0.005)$ up regulation of NF-KB signaling (A, I-1 signaling pathway $(B)$, and Hsp60 and Hsp70 Trr signaling pathways (C). Blue (down-regulation) and red (up-regulation) bars indicate significantly affected genes. The numbers 1-5 represent fold change (treatment vs. control) of gene expression of five independent experiments (i.e. liver slices isolated from 5 mice).

Additional file 9: Table S4. Functions of genes tested by $q-P C R$, source GeneCards http://www.genecards.org.

Additional file 10: Figure S6. A-B. Effects of cholestasis on the expression of genes involved in Fxr-regulated pathways related to lipid and bile acids metabolism in human liver biopsies. Both biliary atresia and intrahepatic cholestasis significantly down-regulated Bile acids regulation of glucose and lipid metabolism via Fxr (A), and Fxr-dependent negative-feedback regulation of bile acids concentration (B). Blue and red bars indicate down-and up-regulation respectively of significantly affected genes. The bar numbers 1 and 2 indicate liver biopsies obtained from patients suffering from biliary atresia and intrahepatic cholestasis, respectively. Each bar represents average fold change of gene expression (disease vs. control) in liver biopsies. For an explanation of the MetaCore symbols is referred to http://pathwaymaps.com/pdf/MC_legend.pdf.

Additional file 11: Figure S7. A. Cell adhesion ECM remodelling pathway is affected in liver biopsies of patients suffering from cholestasis. In two types of human cholestasis, biliary atresia (bar 1) and intrahepatic cholestasis (bar 2) both Cell adhesion_ECM remodelling pathway $(p<0.005)(A)$ was affected. Explanation of the bars is given in the legend of Additional file 10: Figure 56.

Additional file 12: Microarray data from PCLS exposed to cholestatic model compounds. Microarray data were generated using liver slices obtained from livers obtained from 5 mice per one experimental condition. Abbreviations used: M1-5 stands for mouse $1-5$, ctr stands for control, CsA CyclosporinA, CPZ chlorpromazine, 24 means $24 \mathrm{~h}$ incubation time. Data are normalized and presented as $\log 2$ values of intensities.

\section{Competing interests}

The authors declare that they have no competing interests.

\section{Authors' contributions}

ESZ designed the study, performed experiments, analyzed data, wrote the manuscript, GS performed experiments, MG analyzed data, PH and AP analyzed data and edited the manuscript. All authors read and approved the final manuscript.

\section{Acknowledgment and funding}

This work was supported by the Netherlands Genomics Initiative, the Netherlands Organisation for Scientific Research, and the Netherlands Toxicogenomics Centre (grant number 05060510).

We would like to thank to Jan Ossenkoppele for his technical assistance in the histological experiments and Harrie Kools for his help in the processing of raw microarray data.

Received: 1 June 2013 Accepted: 7 October 2013

Published: 10 October 2013

\section{References}

1. Velayudham LS, Farrell GC: Drug-induced cholestasis. Expert Opin Drug Saf 2003, 2(3):287-304

2. Lefebvre $P$, Cariou B, Lien F, Kuipers F, Staels B: Role of bile acids and bile acid receptors in metabolic regulation. Physiol Rev 2009, 89(1):147-191.

3. Dandel M, Lehmkuhl HB, Knosalla C, Hetzer R: Impact of different long-term maintenance immunosuppressive therapy strategies on patients' outcome after heart transplantation. Transpl Immunol 2010, 23(3):93-103.

4. Kienhuis AS, Vitins AP, Pennings JL, Pronk TE, Speksnijder EN, Roodbergen M, van Delft JH, Luijten M, van der Ven LT: Cyclosporine A treated in vitro models induce cholestasis response through comparison of phenotype-directed gene expression analysis of in vivo Cyclosporine A-induced cholestasis. Toxicol Lett 2013, 221(3):225-236.

5. Abernathy CO, Zimmerman HJ, Ishak KG, Utili R, Gillespie J: Drug-induced cholestasis in the perfused rat liver and its reversal by tauroursodeoxycholate: an ultrastructural study. Proc Soc Exp Biol Med 1992, 199(1):54-58

6. Carlton VE, Pawlikowska L, Bull LN: Molecular basis of intrahepatic cholestasis. Ann Med 2004, 36(8):606-617.

7. Setchell KD, Schwarz M, O'Connell NC, Lund EG, Davis DL, Lathe R, Thompson HR, Weslie TR, Sokol RJ, Russell DW: Identification of a new inborn error in bile acid synthesis: mutation of the oxysterol 7alphahydroxylase gene causes severe neonatal liver disease. J Clin Invest 1998 102(9):1690-1703.

8. Hadzic N, Bull LN, Clayton PT, Knisely AS: Diagnosis in bile acid-CoA: amino acid N-acyltransferase deficiency. World J Gastroenterol 2012, 18(25):3322-3326.

9. Zollner $\mathrm{G}$, Wagner $\mathrm{M}$, Trauner M: Nuclear receptors as drug targets in cholestasis and drug-induced hepatotoxicity. Pharmacol Ther 2010, 126(3):228-243

10. Halilbasic E, Claudel T, Trauner M: Bile acid transporters and regulatory nuclear receptors in the liver and beyond. J Hepatol 2013, 58(1):155-68.

11. Kass GE: Mitochondrial involvement in drug-induced hepatic injury. Chem Biol Interact 2006, 163(1-2):145-159.

12. Geier A, Fickert $P$, Trauner M: Mechanisms of disease: mechanisms and clinical implications of cholestasis in sepsis. Nat Clin Pract Gastroenterol Hepatol 2006, 3(10):574-585.

13. Khan AA, Chow EC, Porte RJ, Pang KS, Groothuis GM: The role of lithocholic acid in the regulation of bile acid detoxication, synthesis, and transport proteins in rat and human intestine and liver slices. Toxicolln Vitro 2011, 25(1):80-90.

14. Elferink MG, Olinga P, Draaisma AL, Merema MT, Bauerschmidt S, Polman J, Schoonen WG, Groothuis GM: Microarray analysis in rat liver slices correctly predicts in vivo hepatotoxicity. ToxicolApp/Pharmacol 2008, 229(3):300-309.

15. Silva MF, Aires CC, Luis PB, Ruiter JP, IJIst L, Duran M, Wanders RJ, Tavares de Almeida I: Valproic acid metabolism and its effects on mitochondrial fatty acid oxidation: a review. J Inherit Metab Dis 2008, 31(2):205-216.

16. Vassallo P, Trohman RG: Prescribing amiodarone: an evidence-based review of clinical indications. JAMA 2007, 298(11):1312-1322. 
17. Burk RF, Lawrence RA, Lane JM: Liver necrosis and lipid peroxidation in the rat as the result of paraquat and diquat administration. Effect of selenium deficiency. J Clin Invest 1980, 65(5):1024-1031.

18. Timbrell JA, Mitchell JR, Snodgrass WR, Nelson SD: Isoniazid hepatoxicity: the relationship between covalent binding and metabolism in vivo. J Pharmacol Exp Ther 1980, 213(2):364-369.

19. Groot MJ, Ossenkoppele JS, Bakker R, Pfaffl MW, Meyer HH, Nielen MW Reference histology of veal calf genital and endocrine tissues - an update for screening on hormonal growth promoters. J Vet Med A Physiol Pathol Clin Med 2007, 54(5):238-246.

20. Qu Y, He F, Chen Y: Different effects of the probe summarization algorithms PLIER and RMA on high-level analysis of Affymetrix exon arrays. BMC Bioinformatics 2010, 11:211.

21. Jetten MJ, Gaj S, Ruiz-Aracama A, de Kok TM, van Delft JH, Lommen A, van Someren EP, Jennen DG, Claessen SM, Peijnenburg AA, et al: Omics analysis of low dose acetaminophen intake demonstrates novel response pathways in humans. Toxicol Appl Pharmacol 2012, 259(3):320-328.

22. Subramanian A, Tamayo P, Mootha VK, Mukherjee S, Ebert BL, Gillette MA, Paulovich A, Pomeroy SL, Golub TR, Lander ES, et al: Gene set enrichment analysis: a knowledge-based approach for interpreting genome-wide expression profiles. Proc Natl Acad Sci U S A 2005, 102(43):15545-15550.

23. Jelier R, Schuemie MJ, Veldhoven A, Dorssers LC, Jenster G, Kors JA: Anni 2.0: a multipurpose text-mining tool for the life sciences. Genome Biol 2008, 9(6):R96

24. Franceschini A, Szklarczyk D, Frankild S, Kuhn M, Simonovic M, Roth A, Lin J, Minguez $P$, Bork $P$, von Mering $C$, et al: STRING v9.1: protein-protein interaction networks, with increased coverage and integration. Nucleic Acids Res 2013, 41(Database issue):D808-815.

25. Livak KJ, Schmittgen TD: Analysis of relative gene expression data using real-time quantitative PCR and the 2(-Delta Delta C(T)) Method. Methods 2001, 25(4):402-408.

26. van Summeren A, Renes J, Bouwman FG, Noben JP, van Delft JH, Kleinjans JC, Mariman EC: Proteomics investigations of drug-induced hepatotoxicity in HepG2 cells. Toxicol Sci 2011, 120(1):109-122

27. Gray S, Wang B, Orihuela Y, Hong EG, Fisch S, Haldar S, Cline GW, Kim JK, Peroni OD, Kahn BB, et al: Regulation of gluconeogenesis by Kruppel-like factor 15. Cell Metab 2007, 5(4):305-312.

28. Renga B, Mencarelli A, D'Amore C, Cipriani S, Baldelli F, Zampella A, Distrutti E, Fiorucci S: Glucocorticoid receptor mediates the gluconeogenic activity of the farnesoid $X$ receptor in the fasting condition. FASEB J 2012, 26(7):3021-3031.

29. Trauner M, Claudel T, Fickert P, Moustafa T, Wagner M: Bile acids as regulators of hepatic lipid and glucose metabolism. J Dig Dis 2010, 28(1):220-224.

30. Barth A, Braun J, Muller D: Influence of Verapamil and Cyclosporin A on bile acid metabolism and transport in rat liver slices. Exp Toxicol Pathol 2006, 58(1):31-37.

31. Van Dyke RW, Scharschmidt BF: Effects of chlorpromazine on $\mathrm{Na}+-\mathrm{K}+-$ ATPase pumping and solute transport in rat hepatocytes. Am J Physiol 1987, 253(5 Pt 1):G613-G621.

32. Hadi M, Dragovic S, van Swelm R, Herpers B, van de Water B, Russel FG, Commandeur JN, Groothuis GM: AMAP, the alleged non-toxic isomer of acetaminophen, is toxic in rat and human liver. Arch Toxicol 2013, 87(1):155-165

33. Jemnitz K, Veres Z, Monostory K, Kobori L, Vereczkey L: Interspecies differences in acetaminophen sensitivity of human, rat, and mouse primary hepatocytes. Toxicol In Vitro 2008, 22(4):961-967.

34. Chanussot F, Benkoel L: Prevention by dietary ( $n-6)$ polyunsaturated phosphatidylcholines of intrahepatic cholestasis induced by cyclosporine A in animals. Life Sci 2003, 73(4):381-392.

35. Antherieu S, Bachour-El Azzi P, Dumont J, Abdel-Razzak Z, Guguen-Guillouzo C, Fromenty B, Robin MA, Guillouzo A: Oxidative stress plays a major role in chlorpromazine-induced cholestasis in human HepaRG cells. Hepatology 2013, 57(4):1518-1529.

36. Sinal CJ, Tohkin M, Miyata M, Ward JM, Lambert G, Gonzalez FJ: Targeted disruption of the nuclear receptor FXR/BAR impairs bile acid and lipid homeostasis. Cell 2000, 102(6):731-744.

37. Fujimura $\mathrm{H}$, Murakami $\mathrm{N}$, Kurabe $\mathrm{M}$, Toriumi $\mathrm{W}$ : In vitro assay for drug-induced hepatosteatosis using rat primary hepatocytes, a fluorescent lipid analog and gene expression analysis. J App/ Toxicol 2009, 29(4):356-363.
38. Delgado TC, Barosa C, Nunes PM, Scott DK, O'Doherty RM, Cerdan S, Geraldes CF, Jones JG: Effect of cyclosporine A on hepatic carbohydrate metabolism and hepatic gene expression in rat. Expert Opin Drug Metab Toxicol 2012, 8(10):1223-1230.

39. Shin M, Asada S, Mizumori N, Sano K, Umezawa C: Effect of thioridazine or chlorpromazine on increased hepatic NAD + level in rats fed clofibrate, a hypolipidaemic drug. J Pharm Pharmacol 1998, 50(4):431-436.

40. Hoshi K, Fujino S: Difference between effects of chlorpromazine and perphenazine on microsomal phospholipids and enzyme activities in rat liver. J Toxicol Sci 1992, 17(2):69-79.

41. Ding $Z$, Kng $Y$, Yang H, Ke Z, Zhuo L: An orally available small imidazolium salt ameliorates inflammation and fibrosis in a murine model of cholestasis. Lab Invest 2011, 91(5):752-763.

42. Rezzani R: Exploring cyclosporine A-side effects and the protective role-played by antioxidants: the morphological and immunohistochemical studies. Histol Histopathol 2006, 21(3):301-316.

43. Wolf A, Trendelenburg CF, ez-Fernandez C, Prieto P, Houy S, Trommer WE, Cordier A: Cyclosporine A-induced oxidative stress in rat hepatocytes. J Pharmacol Exp Ther 1997, 280(3):1328-1334.

44. Kitamura M: Control of NF-kappaB and inflammation by the unfolded protein response. Int Rev Immunol 2011, 30(1):4-15.

45. Oo YH, Shetty S, Adams DH: The role of chemokines in the recruitment of lymphocytes to the liver. Dig Dis 2010, 28(1):31-44.

46. Nascimbeni M, Bourdoncle P, Penna C, Saunier B: Recruitment and interaction of human dendritic and $T$ cells in autologous liver slices experimentally infected with HCV produced in cell culture. J Immunol Methods 2012, 378(1-2):51-55.

47. De Maio A: Heat shock proteins: facts, thoughts, and dreams. Shock 1999, 11(1):1-12.

48. Johnson $A E$, van Waes MA: The translocon: a dynamic gateway at the ER membrane. Annu Rev Cell Dev Biol 1999, 15:799-842.

49. Ryffel B, Foxwell BM, Gee A, Greiner B, Woerly G, Mihatsch MJ: Cyclosporine-relationship of side effects to mode of action Transplantation 1988, 46(2 Suppl):90S-96S.

50. Roman ID, Monte MJ, Gonzalez-Buitrago JM, Esteller A, Jimenez R: Inhibition of hepatocytary vesicular transport by cyclosporin $A$ in the rat: relationship with cholestasis and hyperbilirubinemia. Hepatology 1990 12(1):83-91

51. Veel T, Villanger $\mathrm{O}$, Holthe MR, Skjorten FS, Raeder MG: Intravenous bilirubin infusion causes vacuolization of the cytoplasm of hepatocytes and canalicular cholestasis. Acta Physio/ Scand 1991, 143(4):421-429.

52. Kim MS, Shigenaga J, Moser A, Feingold K, Grunfeld C: Repression of farnesoid $X$ receptor during the acute phase response. J Biol Chem 2003 278(11):8988-8995.

53. Kim MS, Sweeney TR, Shigenaga JK, Chui LG, Moser A, Grunfeld C, Feingold KR: Tumor necrosis factor and interleukin 1 decrease RXRalpha, PPARalpha, PPARgamma, LXRalpha, and the coactivators SRC-1, PGC-1alpha, and PGC-1beta in liver cells. Metabolism 2007, 56(2):267-279.

54. Auli M, Domenech A, Andres A, Orta M, Salva M, Descotes J, Prats N: Multiparametric immunotoxicity screening in mice during early drug development. Toxicol Lett 2012, 214(2):200-208.

55. Liangpunsakul S, Chalasani N: Treatment of Nonalcoholic Fatty Liver Disease. Curr Treat Options Gastroenterol 2003, 6(6):455-463.

56. Yip WW, Burt AD: Alcoholic liver disease. Semin Diagn Pathol 2006, 23(3-4):149-160

57. Dulmovits $B M$, Herman IM: Microvascular remodeling and wound healing: a role for pericytes. Int J Biochem Cell Biol 2012, 44(11):1800-1812.

58. Kim WR, Brown RS Jr, Terrault NA, El-Serag H: Burden of liver disease in the United States: summary of a workshop. Hepatology 2002, 36(1):227-242.

doi:10.1186/1755-8794-6-39

Cite this article as: Szalowska et al:: Treatment of mouse liver slices with cholestatic hepatotoxicants results in down-regulation of Fxr and its target genes. BMC Medical Genomics 2013 6:39. 